\title{
SHEAF CONSTRUCTIONS AND THEIR ELEMENTARY PROPERTIES
}

BY

STANLEY BURRIS AND HEINRICH WERNER

\begin{abstract}
We are interested in sheaf constructions in model-theory, so an attempt is made to unify and generalize the results to date, namely various forms of the Feferman-Vaught Theorem, positive decidability results, and constructions of model companions. The task is considerably simplified by introducing a new definition of sheaf constructions over Boolean spaces.
\end{abstract}

We start the paper by giving a new formulation in $\$ 1$ of what sheaf constructions over Boolean spaces are-perhaps an appropriate name for our construction would be 'Boolean product'. In $\$ 2$ we show that this construction is closely related to the formation of reduced products, in particular the universal Horn class generated by a class is the class of structures which can be embedded into the Boolean products of the given class. A specialized Boolean product operator $\Gamma^{e}$ is indeed remarkably similar to reduced products, for in $\$ 3$ it is shown that $\Gamma^{e}$ preserves a sentence iff it is equivalent to a Horn sentence; and in $\$ 4$ we take another look at Comer's version of the Feferman and Vaught Theorem (for sheaves) and show that it is the most general of those currently in use. Several model-theoretic results proved by Waszkiewicz and Weglorz for reduced limit powers have natural extensions to Boolean products as one also sees in $\S 4$.

In $\$ 5$ we take a construction introduced by Arens and Kaplansky, which we call a filtered Boolean power, and show how to extend a translation introduced by Ershov to such structures-this translation is a considerable simplification of the Feferman and Vaught approach of $\$ 4$. Then in $\$ 6$ we prove the result which motivated us to look at the elementary properties of sheaf constructions, namely that countable Boolean products of finitely many finite structures with a finite language have a decidable theory. This generalizes Comer's results for monadic algebras and $\left(x^{m}=x\right)$-rings, and Ershov's results for bounded Boolean powers.

The next section contains technical results needed for the study of model

Received by the editors September 17, 1976 and, in revised form, February 8, 1977.

AMS (MOS) subject classifications (1970). Primary 02H13, 02G05; Secondary 02G10, 02G15, 02G20, 08A20.

Key words and phrases. Subdirect product, Boolean space, Łos's Theorem, preservation theorem, reduced product, filtered Boolean power, decidable theory, discriminator variety, model companion, universal Horn class. 
companions. Then in $\S 8$ it is shown that if a class $\Re$ has few existential $n$-types for $n<\omega$ then the universal Horn class generated by $\Re$ has a model companion. An easy consequence of this is that the universal Horn class generated by finitely many finite structures always has a model companion. In $\$ 9$ the sheaf representation theorem of Bulman-Fleming, Keimel and Werner, for discriminator varieties is used to show that a discriminator variety generated by an elementary class of simple algebras with a modelcomplete theory has an elementary class of algebraically closed members, and a model companion. Axiom systems are given as well as structure theorems for members of these classes. Also some examples of residually large varieties with decidable theories are constructed. Then in $\$ 10$ the results of $\$ 9$ are generalized to include the results of Macintyre and Comer on model-completeness for structures of sheaves. This paper is a considerable revision of preprints [7].

ACKNOWLEDGEMENTS. $\S \S 5$ and 6 were completed while the second author was a Visiting Research Mathematician in the Department of Pure Mathematics at the University of Waterloo during January-March of 1975, a visit made possible by the Deutsche Forschungsgemeinschaft, and the University of Waterloo. We are grateful to Comer for providing us at this time with his recent preprints on monadic algebras. The remaining sections were developed while the first author was a Gastforscher at the Technische Hochschule Darmstadt from May till November, 1975-this research was sponsored by NRC, the DAAD, and primarily the Alexander von Humboldt Foundation. The concept of Boolean product was inspired by recent papers of Volger and Weispfennig on Boolean-valued structures. For $\$ \S 8-10$ we are indebted to some most helpful conversations with Macintyre and Sabbagh at the International Mathematical Summer Center session on "Model Theory and its Applications" in Bressanone during June, 1975. Most of the results of this paper have been presented in the Mittagsseminar directed by Burmeister, Keimel, and Wille-their constructive criticism was very much appreciated. $\$ 8$ was put in its final form, following a suggestion of Alan Adamson, during a seminar given by McKenzie and the first author on "Model Companions of Varieties" at Berkeley in the Winter term of 1975-1976.

1. The operators $\Gamma^{a}$ and $\Gamma^{e}$. For notational simplicity we use capital italic letters $A, B, \ldots$ for structures as well as their universes. For most of the text we will consider only two types of first-order structures, namely Boolean algebras and structures for a given language $L$ (involving possibly both function and relation symbols) with equality. When we speak of a structure we will mean a structure for $L$, and when we speak of a formula we will mean a formula in the language $L$-unless explicitly stated otherwise. Our first-order logical symbols are $\vee, \&, \neg, \exists, \forall, \rightarrow, \leftrightarrow$, and finite conjuncts, respectively 
disjuncts, are denoted by $\&$, respectively $\bigvee$. The Boolean algebra operations are $\vee, \wedge,-, 0,1$, where "-" is relative difference, and finite meets, respectively joins, will be denoted by $\wedge$, respectively $\bigvee$. If $I$ is a set then $P(I)$ denotes the Boolean algebra of subsets of $I$. A theory $T$ is a deductively closed set of sentences. If $\Omega$ is a class of structures then $\operatorname{Th}(\Omega)$ is the theory of $\Re$, i.e. the set of all sentences true of $\Re$. We use $\equiv$ for elementary equivalence and $\prec$ for elementary substructure.

The reader needs to know only the elements of Stone duality between Boolean algebras and Boolean spaces (see [22]). If $B$ is a Boolean algebra let $B^{*}$ be the associated Boolean space of ultrafilters in $B$ (with a basis of clopen sets of the form $\left\{\mathscr{U} \in B^{*} \mid b \in \mathcal{U}\right\}$, where $b \in B$ ); and if $X$ is a Boolean space (i.e. a totally disconnected compact Hausdorff space) let $X^{*}$ be the Boolean algebra of clopen subsets of $X$. Then $B$ is isomorphic to $B^{* *}$ under the map $b \mapsto\left\{\mathscr{Q} \in B^{*} \mid b \in \mathcal{U}\right\}$, and $X$ is homeomorphic to $X^{* *}$ under $x \mapsto\left\{Y \in X^{*} \mid x \in Y\right\}$. We will use capital italic letters $X, Y, \ldots$ at the end of the alphabet for Boolean spaces (and closed subsets of Boolean spaces).

A structure $A$ is a subdirect product of structures $A_{i}, i \in I$, if (i) $A$ is a subset of $\Pi_{i \in I} A_{i}$, (ii) for any atomic formula $\Phi\left(u_{1}, \ldots, u_{m}\right)$ and $f_{1}, \ldots, f_{m} \in A$ we have $A=\Phi\left(f_{1}, \ldots, f_{m}\right)$ iff $A_{i} \vDash \Phi\left(f_{1}(i), \ldots, f_{m}(i)\right)$ for $i \in I$, and (iii) the projection maps $\pi_{i}: \prod_{i \in I} A_{i} \rightarrow A_{i}$ map $A$ onto $A_{i}$ for $i \in I$. If $A$ is a subdirect product of $A_{i}$ for $i \in I$ and $\Phi\left(u_{1}, \ldots, u_{m}\right)$ is a formula then for $f_{1}, \ldots, f_{m} \in$ $A$ we define $\left[\Phi\left(f_{1}, \ldots, f_{m}\right)\right]_{A}$ to be $\left\{i \in I \mid A_{i} \vDash \Phi\left(f_{1}(i), \ldots, f_{m}(i)\right)\right\}$. Usually we will write just $\left[\Phi\left(f_{1}, \ldots, f_{m}\right)\right]$ because, if nothing else, the parameters $f_{1}, \ldots, f_{m}$ will make it clear which structure is meant.

If $\Re$ is a class of structures, then $A \in \Gamma^{a}(\Re)$ means that there is a Boolean space $X(A)$ and structures $A_{x}$ in $\Re$ for $x \in X(A)$ such that

(1) $A$ is a subdirect product of the $A_{x}$,

$\left(2^{a}\right)$ (Atomic Extension Property) for any atomic formula $\Phi\left(u_{1}, \ldots, u_{m}\right)$ and $f_{1}, \ldots, f_{m} \in A,\left[\Phi\left(f_{1}, \ldots, f_{m}\right)\right]$ is a clopen subset of $X(A)$, and

(3) (Patchwork Property) ${ }^{1}$ for $f, g \in A$ and $N$ a clopen subset of $X(A)$ the function $h$ defined by

$$
h(x)= \begin{cases}f(x) & \text { if } x \in N, \\ g(x) & \text { if } x \in X(A)-N,\end{cases}
$$

is in $A .^{2}$

Letting $f \uparrow_{N}$ denote the restriction of $f$ to $N$, etc., we can also write $h=f \uparrow_{N} \cup g \uparrow_{X(A)-N}$, a notation which we will use frequently. Note that if $L$

\footnotetext{
${ }^{1}$ The Patchwork Property appears in Weispfennig [41].

2 To be more precise we should say that there is a set $X(A)$ which can be endowed with a topology $T$ such that $(X(A), T)$ is a Boolean space and (1)-(3) hold.
} 
is a language of algebras then we can replace the $\Phi\left(u_{1}, \ldots, u_{m}\right)$ in condition $\left(2^{a}\right)$ by $u_{1}=u_{2}$, i.e. we require only that equalizers $\left[f_{1}=f_{2}\right]$ be clopen. If in $\left(2^{a}\right)$ we replace "clopen" by "open" we obtain (up to isomorphism), with (1) and (3), exactly what is meant by "all structures of continuous sections of sheaves over Boolean spaces with stalks from $\Re$ ". This is discussed in detail in the Appendix.

There is an important restriction of $\Gamma^{a}$, which we shall call $\Gamma^{e}$, obtained by replacing $\left(2^{a}\right)$ by the following:

$\left(2^{e}\right)$ (Elementary Extension Property) for every formula $\Phi\left(u_{1}, \ldots, u_{m}\right)$ and $f_{1}, \ldots, f_{m} \in A$, the set $\left[\Phi\left(f_{1}, \ldots, f_{m}\right)\right]$ is a clopen subset of $X(A)$.

This condition was introduced by Comer and leads to the following fundamental property.

Lemma 1.1 (The Maximal Property for $\Gamma^{e}$ ). ${ }^{3}$ If $A \in \Gamma^{e}(\Re)$ and $\Phi\left(u, u_{1}, \ldots, u_{m}\right)$ is a formula, then for $f_{1}, \ldots, f_{m} \in A$ there is an $f \in A$ such that $\left[\exists u \Phi\left(u, f_{1}, \ldots, f_{m}\right)\right]=\left[\Phi\left(f, f_{1}, \ldots, f_{m}\right)\right]$.

Proof. For each $x \in Y=\left[\exists u \Phi\left(u, f_{1}, \ldots, f_{m}\right)\right]$ choose an $f_{x} \in A$ such that $x \in\left[\Phi\left(f_{x}, f_{1}, \ldots, f_{m}\right)\right]$ (this is possible by (1)). From $\left(2^{e}\right)$ we see that $\left\{\left[\Phi\left(f_{x}\right.\right.\right.$, $\left.\left.\left.f_{1}, \ldots, f_{m}\right)\right] \mid x \in Y\right\}$ is a clopen cover of the compact set $\left[\exists u \Phi\left(u, f_{1}, \ldots, f_{m}\right)\right]$, hence we can find, for suitable $k, x_{1}, \ldots, x_{k}$ in the set $\left[\exists u \Phi\left(u, f_{1}, \ldots, f_{m}\right)\right]$ such that $\left\{\left[\Phi\left(f_{x}, f_{1}, \ldots, f_{m}\right)\right] \mid 1 \leqslant i \leqslant k\right\}$ covers $\left[\exists u \Phi\left(u, f_{1}, \ldots, f_{m}\right)\right]$. If we let $N_{i}$ be the clopen set $\left[\Phi\left(f_{x_{i}}, f_{1}, \ldots, f_{m}\right)\right]-\cup_{1<j<i}\left[\Phi\left(f_{x_{i}}, f_{1}, \ldots, f_{m}\right)\right]$ for $1 \leqslant i \leqslant k$, and let $N_{0}$ be $X(A)-\cup_{1 \leqslant i \leqslant k} N_{i}$, then defining $f$ to be $f_{x_{1}} \uparrow_{N_{0}} \cup$ $\cup_{1 \leqslant i \leqslant k} f_{x_{i}} \uparrow_{N_{i}}$ we have by the Patchwork Property that $f \in A$, and clearly $\left[\Phi\left(f, f_{1}, \ldots, f_{m}\right)\right]=\left[\exists u \Phi\left(u, f_{1}, \ldots, f_{m}\right)\right]$.

2. Basic results on class operators. We will be interested in the following list of operators:

$\mathbf{I}=$ closure under isomorphism,

$\mathbf{S}=$ closure under substructure,

$\mathbf{S}^{(<)}=$closure under elementary substructure,

$\mathbf{P}=$ closure under direct product,

$\mathbf{P}_{S}=$ closure under subdirect product,

$\mathbf{P}_{R}=$ closure under reduced product (filter product),

$\mathbf{P}_{U}=$ closure under ultraproduct,

$\hat{\mathbf{P}}_{L R}=$ closure under limit reduced power, and

$\hat{\mathbf{P}}_{L U}=$ closure under limit ultrapower.

If $\mathbf{O}_{1}$ and $\mathbf{O}_{2}$ are two class operators let $\mathbf{O}_{1} \leqslant \mathbf{O}_{2}$ be defined to mean $\mathbf{O}_{1}(\Omega) \subseteq \mathbf{I O}_{2}(\Re)$ for all $\Re$, and then $\mathbf{O}_{1}=\mathbf{O}_{2}$ means $\mathbf{O}_{1} \leqslant \mathbf{O}_{2}$ and $\mathbf{O}_{2} \leqslant \mathbf{O}_{1}$. If $f \in \Pi_{i \in I} A_{i}$ and $\mathscr{D}$ is a filter on $P(I)$ then $f / \mathscr{D}$ is the element of

\footnotetext{
${ }^{3}$ We are indebted to Volger for pointing out the key role of this property.
} 
$\prod_{i \in I} A_{i} / \mathscr{D}$ corresponding to $f$. It will be convenient to abbreviate $u_{1}, \ldots, u_{m}$ by $\vec{u}, f_{1}, \ldots, f_{m}$ by $\vec{f}, f_{1}, \ldots, f_{m} \in A$ by $\vec{f} \in A, f_{1} / \mathscr{D}, \ldots, f_{m} / \mathcal{D}$ by $\vec{f} / \mathscr{D}$, $\alpha\left(f_{1}\right), \ldots, \alpha\left(f_{m}\right)$ by $\alpha(\vec{f})$, and $\exists u_{1} \ldots \exists u_{m}$ by $\exists \vec{u}$.

We need Łos's Theorem on ultraproducts in the following, namely if $\mathscr{Q}$ is an ultrafilter on an index set $I, \Phi(\vec{u})$ is a formula, and $\vec{f}$ is in $\Pi_{i \in I} A_{i}$, then $\prod_{i \in I} A_{i} / \mathcal{U}=\Phi(\vec{f} / \mathcal{Q})$ iff $[\Phi(\vec{f})] \in \mathcal{Q}$.

THEOREM 2.1. (a) I $\leqslant \Gamma^{e} \leqslant \Gamma^{a} \leqslant \mathbf{P}_{S} \leqslant \mathrm{SP}$,

(b) $\mathbf{P}_{R} \leqslant \Gamma^{e} \mathbf{P}_{U}$,

(c) $\hat{\mathbf{P}}_{L R} \leqslant \Gamma^{e} \hat{\mathbf{P}}_{L U}$, and

(d) $\mathbf{S P}_{R}=\mathbf{S P P}_{U}=\mathbf{S \Gamma}^{e} \mathbf{P}_{U}=\mathbf{S \Gamma}^{a} \mathbf{P}_{U}{ }^{4}$

Proof. (a) (Obvious.)

(b) Let the reduced product $\Pi_{i \in I} A_{i} / \mathcal{D}$ be given and define $\lambda$ to be the canonical epimorphism from $P(I)$ to $P(I) / \mathscr{D}$. Let $X$ be the Boolean space $(P(I) / \mathscr{D})^{*}$ and define $\alpha$ from $\prod_{i \in I} A_{i} / \mathscr{D}$ into $\Pi_{\mathcal{L} \in X}\left(\Pi_{i \in I} A_{i} / \lambda^{-1}(\mathcal{Q})\right)$ by $\alpha(f / \mathcal{D})(\mathcal{Q})=f / \lambda^{-1}(\mathcal{Q})$ for $f \in \Pi_{i \in I} A_{i}$. Clearly $\alpha$ embeds $\Pi_{i \in I} A_{i} / \mathscr{D}$ as a subdirect product of the $\prod_{i \in I} A_{i} / \lambda^{-1}(\mathcal{Q})$, $\mathcal{U} \in X$, so we need to show that $\alpha\left(\prod_{i \in I} A_{i} / \mathscr{Q}\right)$ satisfies $\left(2^{e}\right)$ and (3). Note that $\lambda^{-1}(\mathscr{Q})$ is an ultrafilter in $P(I)$ for $\mathscr{Q} \in X$.

So let $\Phi(\vec{u})$ be a formula and suppose $\vec{f} \in \prod_{i \in I} A_{i}$. Then, for $\mathscr{Q} \in X$, we have $\mathcal{Q} \in[\Phi(\alpha(\vec{f} / \mathscr{D}))]$ iff $[\Phi(\vec{f})] \in \lambda^{-1}(\mathcal{Q})$ (this is just Łos's Theorem) iff $[\Phi(\vec{f})] / \mathscr{D} \in \mathcal{Q}$, hence $\left(2^{e}\right)$ holds. Finally, let $f, g \in \Pi_{i \in I} A_{i}$, and let $N$ be a clopen subset of $X$. Then, for some $J \subseteq I, N=\{\mathscr{U} \in X \mid J / \mathscr{D} \in \mathcal{Q}\}$. Let $h=f \uparrow_{J} \cup g \uparrow_{I-J}$. Since $J \subseteq[h=f]$ it follows that for $\mathscr{U} \in N,[h=f] / \mathcal{D}$ $\in \mathscr{U}$, hence $\mathscr{U} \in[\alpha(h / \mathscr{D})=\alpha(f / \mathscr{D})]$. Likewise, if $\mathscr{U} \in X-N$ then $\mathscr{U}$ $\in[\alpha(h / \mathscr{D})=\alpha(g / \mathscr{D})]$, so $\alpha(h / \mathscr{Q})=\alpha(f / \mathscr{D}) \uparrow_{N} \cup \alpha(g / \mathscr{D}) \uparrow_{X-N}$.

This finishes the proof of $(b)$; however we make note of the following result which will be used in the proof of Corollary 4.2. Let $\nu$ be the canonical isomorphism from $P(I) / \mathscr{D}$ onto $(P(I) / \mathscr{D})^{* *}$ given by $\nu(J / \mathcal{D})=\{\mathcal{Q} \in$ $\left.(P(I) / \mathscr{D})^{*} \mid J / \mathscr{D} \in \mathcal{Q}\right\}$, for $J \in P(I)$. Then given a formula $\Phi(\vec{u})$ and parameters $\vec{f} \in \Pi_{i \in I} A_{i}, \nu([\Phi(\vec{f})] / \mathscr{D})=[\Phi(\alpha(\vec{f} / \mathscr{D}))]$.

(c) If $A$ is a structure, $I$ an index set, $\mathscr{F}$ a filter on $I \times I$, and $\mathscr{D}$ a filter on $I$, then the limit power $A^{I} \mid \mathcal{F}$ is the substructure of $A^{I}$ given by $\{f \in$ $\left.A^{I} \mid \operatorname{Ker}(f) \in \mathscr{F}\right\}$, where $\operatorname{Ker}(f)=\{\langle i, j\rangle \in I \times I \mid f(i)=f(j)\}$ and the limit reduced power $A_{\mathscr{D}}^{I} \mid \mathscr{F}$ is the substructure of $A^{I} / \mathscr{D}$ given by $\{f / \mathscr{D} \mid f \in$ $\left.A^{I} \mid \mathscr{F}\right\}$. If $\mathscr{D}$ is an ultrafilter then $A_{\mathscr{D}}^{I} \mid \mathscr{F}$ is a limit ultrapower. Let $P(I) \mid \mathscr{F}$ be $\left\{J \in P(I) \mid J^{2} \cup(I-J)^{2} \in \mathscr{F}\right\}$, and $P(I)_{\mathscr{D}} \mid \mathscr{F}=\{J / \mathscr{D}|J \in P(I)| \mathscr{F}\}$. Let $\lambda$ be the canonical epimorphism from $P(I) \mid \mathscr{F}$ to $P(I)_{\mathscr{D}} \mid \mathscr{F}$, and set $X=$ $\left(P(I)_{\mathscr{D}} \mid \mathscr{F}\right)^{*}$. If $\mathscr{U} \in X$ then $\lambda^{-1}(\mathscr{U})$ is an ultrafilter in $P(I) \mid \mathscr{F}$. For each $\mathscr{U} \in X$ let $\rho(\mathcal{Q})$ be a fixed ultrafilter in $P(I)$ containing $\lambda^{-1}(\mathcal{U})$, and define

${ }^{4}$ The result $\mathbf{S P}_{R}=\mathbf{S P P}_{U}$ is due to Mal'cev [29]. 


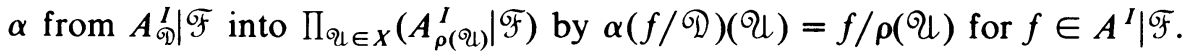
Again it is not difficult to verify that $\alpha$ embeds $A_{\mathscr{D}}^{I} \mid \mathscr{F}$ as a subdirect product of the $A_{\rho(\mathscr{U})}^{I} \mid \mathscr{F}, \mathscr{U} \in X$, so we are left with showing that $\alpha\left(A_{\mathscr{D}}^{I} \mid \mathscr{F}\right)$ satisfies $\left(2^{e}\right)$ and (3).

Let $\Phi(\vec{u})$ be a formula and let $\vec{f}$ be parameters from $A^{I} \mid \mathcal{F}$. Then, for Q $\in X, \mathcal{Q} \in[\Phi(\alpha(\vec{f} / \mathscr{D}))]$ iff $A_{\rho(\mathcal{Q})}^{I} \mid \mathcal{F} \vDash \Phi(\vec{f} / \rho(\mathcal{Q}))$ iff $[\Phi(\vec{f})] \in \rho(\mathcal{Q})$. Now, since $[\Phi(\vec{f})]^{2} \cup(I-[\Phi(\vec{f})])^{2}$ contains $\bigcap_{1 \leqslant i \leqslant m} \operatorname{Ker}\left(f_{i}\right)$ it follows that $[\Phi(\vec{f})] \in$ $P(I) \mid \mathscr{F}$, hence $[\Phi(\vec{f})] \in \rho(\mathcal{U})$ iff $[\Phi(\vec{f})] \in \lambda^{-1}(\mathcal{Q})$, and thus iff $[\Phi(\vec{f})] / \mathscr{D} \in$ $\mathcal{Q}$, so we have proved that $[\Phi(\alpha(\vec{f} / \mathscr{D}))]=\{\mathcal{Q} \in X \mid[\Phi(\vec{f})] / \mathscr{D} \in \mathcal{Q}\}$, hence $\left(2^{e}\right)$ holds. For (3) suppose $f, g \in A^{I} \mid \mathscr{F}$ and $N \in X^{*}$. Then for some $J \in P(I) \mid \mathscr{F}, N=\{\mathcal{Q} \in X \mid J / \mathscr{D} \in \mathcal{U}\}$. Let $h=f \uparrow_{J} \cup g \uparrow_{I-J}$. Then $\operatorname{Ker}(h)$ contains $\operatorname{Ker}(f) \cap \operatorname{Ker}(g) \cap\left(J^{2} \cup(I-J)^{2}\right)$, so $h \in A^{I} \mid \mathscr{F}$. Since $J \subseteq[h=$ $f]$ it follows that for $\mathscr{Q} \in N,[h=f] / \mathscr{D} \in \mathcal{Q}$, hence $\mathscr{Q} \in[\alpha(h / \mathscr{D})=$ $\alpha(f / \mathscr{D})]$, and likewise if $\mathscr{Q} \in X-N$ then $\mathcal{Q} \in[\alpha(h / \mathscr{D})=\alpha(g / \mathscr{D})]$, so $\alpha(h / \mathscr{D})=\alpha(f / \mathscr{D}) \uparrow_{N} \cup \alpha(g / \mathscr{D}) \uparrow_{X-N}$.

This finishes the proof of (c), but we wish to note the following which will be used in the proof of Corollary 4.3. Let $\nu$ be the canonical isomorphism from $P(I)_{\mathscr{D}} \mid \mathscr{F}$ onto $\left(P(I)_{\mathscr{D}} \mid \mathscr{F}\right)^{* *}$ defined by $\nu(J / \mathscr{D})=\{\mathscr{Q} \in$ $\left.\left(P(I)_{\mathscr{D}} \mid \mathscr{F}\right)^{*} \mid J / \mathscr{D} \in \mathcal{Q}\right\}$, where $J \in P(I) \mid \mathscr{F}$. Then, for any formula $\Phi(\vec{u})$ and parameters $\vec{f}$ from $A^{I} \mid \mathscr{F}, \quad \nu([\Phi(\vec{f})] / \mathscr{D})=[\Phi(\alpha(\vec{f} / \mathscr{D}))]$.

(d) We have $\mathbf{S P}_{R} \leqslant \mathbf{S} \Gamma^{e} \mathbf{P}_{U} \leqslant \mathbf{S} \Gamma^{a} \mathbf{P}_{U} \leqslant \mathbf{S}(\mathbf{S P}) \mathbf{P}_{U}=\mathbf{S P P}_{U} \leqslant \mathbf{S} \mathbf{P}_{R}$.

Now we have a good number of examples of Boolean products. Let us look at one more well-known construction, that of a bounded Boolean power. Let $A$ be a structure and $X$ a Boolean space. Then $A[X]^{*}$ is the substructure of the direct power $A^{X}$ with universe $\left\{f \in A^{X} \mid f^{-1}(a) \in X^{*}\right.$ for $\left.a \in A\right\}$. Clearly (1) and (3) are satisfied. For $a \in A$ let $c_{a}$ denote the constant function in $A[X]^{*}$ with value $a$. Then, given a formula $\Phi(\vec{u})$ and $\vec{f} \in A[X]^{*}$, if $x \in[\Phi(\vec{f})]$ then

$$
\bigcap_{1<i<m}\left[f_{i}=c_{f_{i}(x)}\right] \subseteq[\Phi(\vec{f})],
$$

hence $\left(2^{e}\right)$ holds, so $A[X]^{*} \in \Gamma^{e}(\{A\})$.

3. The preservation theorem for $\Gamma^{e}$. We are interested in characterizing those sentences preserved by $\Gamma^{e}$, i.e. sentences $\Phi$ such that if $A \in \Gamma^{e}(\Re)$ then $[\Phi]=X(A)$ implies $A \vDash \Phi$. Recall that a Horn formula is any formula of the form

$$
Q_{1} u_{1} \cdots Q_{n} u_{n} \underset{1<i<k}{\&}\left(\Phi_{i, 1} \vee \cdots \vee \Phi_{i, n_{i}}\right)
$$

where each $\Phi_{i, j}$ is an atomic or the negation of an atomic formula, and for each $i, 1 \leqslant i \leqslant k$, at most one of $\Phi_{i, 1}, \ldots, \Phi_{i, n_{i}}$ is atomic, and the $Q_{1}, \ldots, Q_{n}$ are quantifiers. A Horn sentence is a Horn formula with no free variables. 
TheOREM 3.1. $\Gamma^{e}$ preserves a sentence $\Phi$ iff $\Phi$ is equivalent to a Horn sentence.

Proof. Let $\Phi(\vec{u})$ be a Horn formula and let $A \in \Gamma^{e}(\Omega)$. We will prove by induction on the complexity of $\Phi$ that, for $\vec{f} \in A,[\Phi(\vec{f})]=X(A)$ implies $A \vDash \Phi(\vec{f})$.

If $\Phi(\vec{u})$ is an atomic formula then, by definition, $A \vDash \Phi(\vec{f})$ iff $[\Phi(\vec{f})]=$ $X(A)$, and hence $A \models \neg \Phi(\vec{f})$ if $[\neg \Phi(\vec{f})] \neq \varnothing$. From this it is easy to argue the case that $\Phi$ is an open Horn formula. So now assume that we have verified the claim for a given Horn formula $\Phi(u, \vec{u})$. If $[\exists u \Phi(u, \vec{f})]=X(A)$ then, by the Maximal Property, there is an $f \in A$ with $[\Phi(f, \vec{f})]=X(A)$, and then by assumption $A \models \Phi(f, \vec{f})$, so $A \vDash \exists u \Phi(u, \vec{f})$. If $[\forall u \Phi(u, \vec{f})]=X(A)$ then for any $f \in A,[\Phi(f, \vec{f})]=X(A)$, hence $A \models \Phi(f, \vec{f})$, so $A \models \forall u \Phi(u, \vec{f})$.

Thus we know that Horn sentences are preserved by $\Gamma^{e}$. On the other hand, if a sentence is preserved by $\boldsymbol{\Gamma}^{e}$ then $\mathbf{P}_{R} \leqslant \Gamma^{e} \mathbf{P}_{U}$ ensures that the sentence is preserved by $\mathbf{P}_{R}$, hence it is equivalent to a Horn sentence (see Theorem $6.2 .5^{\prime}$ of $[10$, p. 366]). This gives an alternate proof of the following result which appears in [38].

COROllary 3.2 (WASZKIEWICZ AND Weglorz). $\hat{\mathbf{P}}_{L R}$ preserves a sentence $\Phi$ iff $\Phi$ is equivalent to a disjunct of Horn sentences.

Proof. Since $\hat{\mathbf{P}}_{L R} \leqslant \Gamma^{e} \hat{\mathbf{P}}_{L U}$ it is clear that $\hat{\mathbf{P}}_{L R}$ preserves Horn sentences, hence disjuncts of Horn sentences. On the other hand $\hat{\mathbf{P}}_{R} \leqslant \hat{\mathbf{P}}_{L R}, \hat{\mathbf{P}}_{R}$ being the closure under reduced power, so $\hat{\mathbf{P}}_{L R}$ preserves $\Phi$ implies $\hat{\mathbf{P}}_{R}$ preserves $\Phi$, hence $\Phi$ is equivalent to a disjunct of Horn sentences (see [10, p. 366]).

4. The Feferman and Vaught Theorem for $\Gamma^{e}$, and its consequences. Comer [12] adapted the Feferman and Vaught Theorem [21] to sections of sheaves (this was the reason for introducing the condition $\left(2^{e}\right)$ ) and noted that he had generalized their results for direct products. Rather than ask the reader to translate his proof ${ }^{5}$ we give details here (a slight modification of Chang and Keisler's Proposition 6.3.2 of [10, p. 342]) and further applications.

If $\Phi\left(u_{1}, \ldots, u_{m}\right)$ is a formula involving only the extra-logical symbols, the logical connectives, \&, ᄀ, and the quantifier $\exists$ we will give an effective procedure to find a determining sequence $\left\langle\Phi^{*} ; \Psi_{1}, \ldots, \Psi_{l}\right\rangle$, where $\Phi^{*}\left(z_{1}, \ldots, z_{l}\right)$ is a formula in the language of Boolean algebras and $\Psi_{i}\left(u_{1}, \ldots, u_{m}\right)$ is an $L$-formula, $1 \leqslant i \leqslant l$ :

(i) if $\Phi$ is atomic then the determining sequence is $\left\langle z_{1}=1\right.$; $\left.\Phi\right\rangle$;

(ii) if $\Phi$ is determined by $\left\langle\Phi^{*}\left(z_{1}, \ldots, z_{l}\right) ; \Psi_{1}, \ldots, \Psi_{l}\right\rangle$ then $\neg \Phi$ is determined by the sequence

$$
\left\langle-\Phi^{*}\left(1-z_{1}, \ldots, 1-z_{l}\right) ; \neg \Psi_{1}, \ldots, \neg \Psi_{l}\right\rangle ;
$$

${ }^{5}$ Comer's proof uses, of course, the notation of sheaf theory. 
(iii) if $\Phi$ is determined by $\left\langle\Phi^{*} ; \Psi_{1}, \ldots, \Psi_{l}\right\rangle$ and $\Pi$ is determined by $\left\langle\Pi^{*} ; \Psi_{l+1}, \ldots, \Psi_{l+k}\right\rangle$ then $\Psi \& \Psi$ is determined by

$$
\left\langle\Phi^{*}\left(z_{1}, \ldots, z_{l}\right) \& \Pi^{*}\left(z_{l+1}, \ldots, z_{l+k}\right) ; \Psi_{1}, \ldots, \Psi_{l+k}\right\rangle ;
$$

(iv) if $\Phi(u)$ is determined by $\left\langle\Phi^{*} ; \Psi_{1}, \ldots, \Psi_{l}\right\rangle$ then $\exists u \Phi(u)$ is determined by $\left\langle\Pi ; \Theta_{1}, \ldots, \Theta_{l_{0}}\right\rangle$, where $\Pi, \Theta_{1}, \ldots, \Theta_{l_{0}}$ are defined as follows. Let $P(\{1, \ldots, l\})-\{\varnothing\}=\left\{S_{1}, \ldots, S_{l_{0}}\right\}$ with $S_{i}=\{i\}$ for $1 \leqslant i \leqslant l$. For $1 \leqslant$ $j \leqslant l_{0}$ define $\Theta_{j}$ to be $\exists u\left(\&_{i \in S_{j}} \Psi_{i}\right)$. Then let $\Pi\left(z_{1}, \ldots, z_{l_{0}}\right)$ be the formula

$$
\begin{array}{r}
\exists w_{1} \cdots \exists w_{l_{0}}\left[\left(\underset{1<i<l_{0}}{\&} w_{i} \leqslant z_{i}\right) \&\left(\underset{S_{i} \cup S_{j}=S_{k}}{\&} w_{i} \wedge w_{j}=w_{k}\right)\right. \\
\left.\& \Phi^{*}\left(w_{1}, \ldots, w_{l}\right)\right] .
\end{array}
$$

THEOREM 4.1 (COMER). Let $\Phi(\vec{u})$ be a formula with a determining sequence $\left\langle\Phi^{*}\left(z_{1}, \ldots, z_{l}\right) ; \Psi_{1}(\bar{u}), \ldots, \Psi_{l}(\vec{u})\right\rangle$. Then for $A \in \Gamma^{e}(\Re)$, and for $f_{1}, \ldots, f_{m}$ $\in A$,

$$
A \vDash \Phi(\vec{f}) \quad \text { iff } X(A)^{*} \vDash \Phi^{*}\left(\left[\Psi_{1}(\vec{f})\right], \ldots,\left[\Psi_{1}(\vec{f})\right]\right) .
$$

Proof. One proceeds by induction on the complexity of $\Phi$. The atomic case is just the definition of “ $\vDash$ ". The induction steps for $\neg$ and \& are straightforward.

For the step with the existential quantifier let us assume that the theorem holds for $\Phi\left(u, u_{1}, \ldots, u_{m}\right)$ with a determining sequence $\left\langle\Phi^{*} ; \Psi_{1}, \ldots, \Psi_{l}\right\rangle$. Let $\left\langle\Pi ; \Theta_{1}, \ldots, \Theta_{l_{0}}\right\rangle$ be as in (iv).

If $A=\exists u \Phi(u, \vec{f})$ then choose an $f \in A$ such that $A \models \Phi(f, \vec{f})$. For $1 \leqslant j \leqslant$ $l_{0}$ it is obvious that $\left[\&_{i \in S_{j}} \Psi_{i}(f, \vec{f})\right] \subseteq\left[\Theta_{j}(\vec{f})\right]$, that if $S_{i} \cup S_{j}=S_{k}$ then

$$
\left[\underset{t \in S_{i}}{\&} \Psi_{t}(f, \vec{f})\right] \cap\left[\underset{t \in S_{j}}{\&} \Psi_{t}(f, \vec{f})\right]=\left[\underset{t \in S_{k}}{\&} \Psi_{t}(f, \vec{f})\right]
$$

and

$$
X(A)^{*} \vDash \Phi^{*}\left(\left[\underset{i \in S_{1}}{\&} \Psi_{i}(f, \vec{f})\right], \ldots,\left[\underset{i \in S_{l}}{\&} \Psi_{i}(f, \vec{f})\right]\right)
$$

by assumption. Thus $A=\exists u \Phi(u, \vec{f})$ implies

$$
X(A)^{*}=\Pi\left(\left[\Theta_{1}(\vec{f})\right], \ldots,\left[Q_{l_{0}}(\vec{f})\right]\right) .
$$

Conversely, if $X(A)^{*} \vDash \Pi\left(\left[\Theta_{1}(\vec{f})\right], \ldots,\left[\Theta_{l_{0}}(\vec{f})\right]\right)$ then we can find clopen subsets $W_{1}, \ldots, W_{l_{0}}$ such that:

$$
\begin{array}{cl}
X(A)^{*} \vDash W_{i} \subseteq\left[\Theta_{i}(\vec{f})\right] & \text { for } 1 \leqslant i \leqslant l_{0}, \\
X(A)^{*} \vDash W_{i} \cap W_{j}=W_{k} & \text { if } S_{i} \cup S_{j}=S_{k},
\end{array}
$$


and

$$
X(A)^{*} \vDash \Phi^{*}\left(W_{1}, \ldots, W_{l}\right) .
$$

By the Maximal Property we can find $g_{1}, \ldots, g_{l_{0}} \in A$ such that

$$
\left[\exists u \underset{j \in S_{i}}{\&} \Psi_{j}(u, \vec{f})\right]=\left[\&_{j \in S_{i}} \Psi_{j}\left(g_{i}, \vec{f}\right)\right], \quad 1 \leqslant i \leqslant l_{0} .
$$

For each $i, 1 \leqslant i \leqslant l_{0}$, let us define $N_{i}$ to be $W_{i}-\cup\left\{W_{j} \mid S_{i} \subsetneq S_{j}\right\}$, and then let $M=X(A)-\cup_{1 \leqslant i \leqslant l_{0}} N_{i}$. If we now define $g$ to be $\left(\cup_{1 \leqslant i \leqslant l_{0}}^{\neq} g_{i} \uparrow_{N_{i}}\right)$ $\cup g_{1} \uparrow_{M}$, then, for $1 \leqslant i \leqslant l_{0}$ we have the inclusion $W_{i} \subseteq\left[\&_{j \in S_{i}} \Psi_{j}(g, f)\right]$. Since $X(A)^{*} \vDash \Phi^{*}\left(W_{1,}, \ldots, W_{l}\right)$ it follows that $X(A)^{*} \vDash$ $\Phi^{*}\left(\left[\Psi_{1}(g, f)\right], \ldots,\left[\Psi_{l}(g, \vec{f})\right]\right)$ (an easy induction proof shows that the $\Phi^{*}$ of Chang and Keisler is such that $\left[\Phi^{*}\left(z_{1}, \ldots, z_{l}\right) \&\left(\&_{1<i<l} z_{i_{-}} \leqslant z_{i}^{\prime}\right)\right] \rightarrow$ $\Phi^{*}\left(z_{1}^{\prime}, \ldots, z_{l}^{\prime}\right)$ is valid in every Boolean algebra), so $A \models \Phi(g, \vec{f})$, i.e. $A=$ $\exists u \Phi(u, \vec{f})$.

Next we will show that Comer's version of the Feferman and Vaught Theorem contains two other important versions.

Corollary 4.2 (Weinstein). Let $\Phi(\vec{u})$ be a formula with determining sequence $\left\langle\Phi^{*} ; \Psi_{1}, \ldots, \Psi_{l}\right\rangle$. Then, for $\mathcal{D}$ a filter in $P(I)$ and $f_{1}, \ldots, f_{m} \in$ $\prod_{i \in I} A_{i}$,

$$
\begin{aligned}
\prod_{i \in I} A_{i} / \mathscr{D} & =\Phi(\vec{f} / \mathscr{D}) \\
& \text { iff } P(I) / \mathscr{Q} \vDash \Phi^{*}\left(\left[\Psi_{1}(\vec{f})\right] / \mathscr{D}, \ldots,\left[\Psi_{l}(\vec{f})\right] / \mathscr{D}\right) .
\end{aligned}
$$

Proof. Recall the embedding $\alpha: \prod_{i \in I} A_{i} / \mathscr{Q} \rightarrow \prod_{थ \in X}\left(\prod_{i \in I} A_{i} / \lambda^{-1}(\mathscr{Q})\right)$ defined in the proof of Theorem 2.1(b), namely $\alpha(f / \mathscr{D})(\mathcal{Q})=f / \lambda^{-1}(\mathcal{U})$, and the isomorphism $\nu: P(I) / \mathscr{Q} \rightarrow(P(I) / \mathscr{Q}))^{* *}$. Then

$$
\begin{aligned}
& \prod_{i \in I} A_{i} / \mathscr{Q} \vDash \Phi(\vec{f} / \mathscr{D}) \quad \text { iff } \alpha\left(\prod_{i \in I} A_{i} / \mathscr{D}\right) \vDash \Phi(\alpha(\vec{f} / \mathscr{D})) \\
& \quad \text { iff (by Theorem 4.1) } X^{*} \vDash \Phi^{*}\left(\left[\Psi_{1}(\alpha(\vec{f} / \mathscr{D}))\right], \ldots,\left[\Psi_{l}(\alpha(\vec{f} / \mathscr{D}))\right]\right) \\
& \quad \text { iff } P(I) / \mathscr{D} \vDash \Phi^{*}\left(\left[\Psi_{1}(\vec{f})\right] / \mathscr{D}, \ldots,\left[\Psi_{l}(\vec{f})\right] / \mathscr{D}\right)
\end{aligned}
$$

(see the remark at the end of the proof of Theorem 2.1(b)).

Corollary 4.3 (Weglorz, Banaschewski AND Nelson). ${ }^{6}$ Let $\Phi(\vec{u})$ be a formula with a determining sequence $\left\langle\Phi^{*} ; \Psi_{1}, \ldots, \Psi_{l}\right\rangle$. Then, given a limit reduced power $A_{\mathscr{D}}^{I} \mid \mathcal{F}$ and $f_{1}, \ldots, f_{m} \in A^{I} \mid \mathcal{F}$,

${ }^{6}$ Banaschewski and Nelson use this to analyze elementary properties of Boolean powers and bounded Boolean powers in [4]. (Ash has a less general formulation in [2].) Weglorz uses the above to prove a result of Galvin on iterated reduced powers, as well as to show that the free product in Boolean algebras preserves elementary equivalence, in [40]. 


$$
A_{\mathscr{D}}^{I} \mid \mathscr{F} \vDash \Phi(\vec{f} / \mathscr{D}) \quad \text { iff } P(I)_{\mathscr{D}} \mid \mathscr{F} \vDash \Phi^{*}\left(\left[\Psi_{1}(\vec{f})\right] / \mathscr{D}, \ldots,\left[\Psi_{l}(\vec{f})\right] / \mathscr{D}\right) \text {. }
$$

Proof. (Parallel to the proof of Corollary 4.2 using the isomorphisms $\alpha$ and $\nu$ in the proof of Theorem 2.1(c).)

Remark. Comer stated that Theorem 4.1 improves on the Feferman and Vaught Theorem for $\mathbf{P}$, but he makes no mention of it covering the result of Weinstein. Volger [37] has a slight generalization of Theorem 4.1 using Boolean-valued structures. (If one looks only at the global elements of Boolean-valued structures then the results are equivalent.)

Let $\Phi$ be a sentence and $\left\langle\Phi^{*} ; \Psi_{1}, \ldots, \Psi_{l}\right\rangle$ a determining sequence. By putting $\Phi^{*}$ in prenex form with its matrix in disjunctive normal form it is routine to write down a sequence $\left\langle\Phi^{\prime} ; \Theta_{1}, \ldots, \Theta_{2^{\prime}}\right\rangle$ where the $\Theta_{1}, \ldots, \Theta_{2^{\prime}}$ are the various conjuncts $\Sigma_{1} \& \cdots \& \Sigma_{l}, \Sigma_{i}$ being either $\Psi_{i}$ or $\neg \Psi_{i}$, such that for any $A \in \Gamma^{e}(\Re), A \vDash \Phi$ iff $X(A)^{*} \vDash \Phi^{\prime}\left(\left[\Theta_{1}\right], \ldots,\left[\Theta_{2^{\prime}}\right]\right)$. We will refer to this new sequence as a special determining sequence for $\Phi$. Next, if $T$ is any theory of Boolean algebras let $\Gamma_{T}^{e}(\Re)=\left\{A \in \Gamma^{e}(\Re) \mid X(A)^{*} \vDash T\right\}$. The proof of the following is an easy adaptation of methods in Feferman and Vaught.

LEMMA 4.4. Let $\Phi$ be an L-sentence and $\Re$ a class of L-structures. If $\left\langle\Phi^{\prime} ; \Theta_{1}, \ldots, \Theta_{2^{\prime}}\right\rangle$ is a special determining sequence for $\Phi$, let $\Theta_{m+1}, \ldots, \Theta_{2^{\prime}}$ be those $\Theta_{i}$ such that $\Re \vDash \neg \Theta_{i}$ (of course this collection may be empty). Then, for $T$ a theory of Boolean algebras,

$$
\begin{aligned}
& \Gamma_{T}^{e}(\Re) \vDash \Phi \\
& \text { iff } T \vdash \forall z_{1} \cdots \forall z_{m}\left\{\left[z_{1} \vee \cdots \vee z_{m}=1 \& \underset{1<i<j<m}{\&} z_{i} \wedge z_{j}=0\right]\right. \\
& \left.\rightarrow \Phi^{\prime}\left(z_{1}, \ldots, z_{m}, 0, \ldots, 0\right)\right\} \text {. }
\end{aligned}
$$

Proof. Suppose the second condition holds. Then for any $A \in \Gamma_{T}^{e}(\Re)$ the sets $\left[\Theta_{1}\right], \ldots,\left[\Theta_{m}\right]$ form a disjoint cover of $X(A)$, so $X(A)^{*} \vDash$ $\Phi^{\prime}\left(\left[\Theta_{1}\right], \ldots,\left[\Theta_{m}\right], \varnothing, \ldots, \varnothing\right)$, hence $A \vDash \Phi$, so $\Gamma_{T}^{e}(\Re) \vDash \Phi$. For the converse suppose $\Gamma_{T}^{e}(\Re) \vDash \Phi$. Let $B \vDash T$ and $b_{1}, \ldots, b_{m} \in B$ with $b_{1} \vee \cdots \vee b_{m} \vDash$ 1 and $b_{i} \wedge b_{j}=0$ if $1 \leqslant i<j \leqslant m$. Define $X_{i}$ to be $\left\{\mathcal{Q} \in B^{*} \mid b_{i} \in \mathcal{Q}\right\}$, $1 \leqslant i \leqslant m$. Then $X_{1}, \ldots, X_{m}$ are pairwise disjoint clopen sets which cover $B^{*}$. Let $A_{i} \in \Re$ be such that $A_{i} \vDash \Theta_{i}, 1 \leqslant i \leqslant m$ (such structures exist by assumption). Now for $\mathscr{U} \in B^{*}$ define $A_{\mathscr{U}}$ to be that $A_{i}$ such that $b_{i} \in \mathcal{U}$, and let $A$ be the subdirect product of the $A_{\mathscr{U}}$ consisting of those $f \in$ $\mathrm{II}_{\mathscr{Q} \in B^{*}} A_{\mathscr{Q}}$ such that $f \uparrow_{X_{i}}$ is a continuous function from $X_{i}$ to $A_{i}$, where, as usual, $A_{i}$ is given the discrete topology. Then it is not difficult to show that $A \cong \Pi_{1 \leqslant i \leqslant m} A_{i}\left[X_{i}\right]^{*}$, hence $A$ is in $\Gamma_{T}^{e}(\Re)$, and furthermore $\langle X(A)$; $\left.\left[\Theta_{1}\right], \ldots,\left[\Theta_{m}\right]\right\rangle=\left\langle B^{*} ; X_{1}, \ldots, X_{m}\right\rangle$. By hypothesis $A=\Phi$, hence $X(A)^{*} \vDash$ $\Phi^{\prime}\left(\left[\Theta_{1}\right], \ldots,\left[\Theta_{m}\right], \varnothing, \ldots, \varnothing\right)$, so $B=\Phi^{\prime}\left(b_{1}, \ldots, b_{m}, 0, \ldots, 0\right)$. 
ThEOREM 4.5. (a) If $T$ and $\mathrm{Th}(\Omega)$ are decidable then $\operatorname{Th}\left(\Gamma_{T}^{e}(\Omega)\right)$ is decidable.

(b) $\operatorname{Th}(\Re)=\operatorname{Th}\left(\Omega^{\prime}\right)$ implies $\operatorname{Th}\left(\Gamma_{T}^{e}(\Re)\right)=\operatorname{Th}\left(\Gamma_{T}^{e}\left(\Omega^{\prime}\right)\right)$, for any $T$.

(c) If $\mathrm{Th}(\Re)$ is complete then, for any $A \in \Re$,

$$
\operatorname{Th}\left(\Gamma_{T}^{e}(\Omega)\right)=\operatorname{Th}\left(\left\{A[X]^{*} \mid X^{*} \vDash T\right\}\right) .
$$

(d) If $\mathrm{Th}(\Re)$ and $T$ are both complete then, for any $A \in \Re$ and $X^{*} \vDash T$, $\operatorname{Th}\left(\Gamma_{T}^{e}(\Re)\right)=\operatorname{Th}\left(A[X]^{*}\right)$.

(e) $\operatorname{Th}\left(\Gamma_{T}^{e}(\Re)\right)=\operatorname{Th}\left(\left\{\prod_{i \in I} A_{i} / \mathscr{D} \mid A_{i} \in \Re, P(I) / \mathscr{D} \vDash T\right\}\right)$.

(f) If $A$ is finite or $\operatorname{Th}(A)$ is $\aleph_{0}$-categorical, and if $X^{*}$ is finite or $\operatorname{Th}\left(X^{*}\right)$ is $\aleph_{0}$-categorical, then $A[X]^{*}$ is finite or (provided the language $L$ is countable) $\operatorname{Th}\left(A[X]^{*}\right)$ is $\aleph_{0}$-categorical.

Proof. In the following $\left\langle\Phi^{\prime} ; \Theta_{1}, \ldots, \Theta_{2^{\prime}}\right\rangle$ is a special determining sequence for $\Phi$; for parts (b) - (f) it is as in Lemma 4.4.

(a) Given $\Phi$ we can effectively find a special determining sequence as above, and then effectively determine for which of the $\Theta_{i}$ it is true that $\Re \vDash \neg \Theta_{i}$. Then Lemma 4.4 can be used to decide $\Phi$.

(b) For a given $\Phi$ we can use the same sentence in Lemma 4.4 for both $\Re$ and $\Re^{\prime}$ as $\Re \vDash \neg \Theta_{i}$ iff $\Re^{\prime} \vDash \neg \Theta_{i}$.

(c) In this case, for $A \in \Gamma^{e}(\Re)$, the completeness of $\mathrm{Th}(\Re)$ leads to $A \vDash \Phi$ iff $X(A)^{*} \vDash \Phi^{\prime}(X(A), \varnothing, \ldots, \varnothing)$, so the choice of the individual $A_{x} \in \Omega$ plays no role in the elementary properties of $A$.

(d) (Just an extension of the argument in (c).)

(e) In the proof of Theorem 2.1(b) it is clear that $\mathrm{II}_{i \in I} A_{i} / \mathcal{Q D}$ is in $\mathbf{I \Gamma}_{T}^{e} \mathbf{P}_{U}\left(\left\{A_{i} \mid i \in I\right\}\right)$ if $P(I) / \mathscr{D} \vDash T$, and hence we have, from (b),

$$
\operatorname{Th}\left(\Gamma_{T}^{e}(\Re)\right) \subseteq \operatorname{Th}\left(\left\{\prod_{i \in I} A_{i} / \mathscr{D} \mid A_{i} \in \mathfrak{R}, P(I) / \mathscr{D} \vDash T\right\}\right) .
$$

On the other hand, if $\Gamma_{T}^{e}(\Re) \not \neq \Phi$ let $A \in \Gamma_{T}^{e}(\Re)$ be such that $A \vDash \neg \Phi$, and then let $X_{i}=\left[\Theta_{i}\right]$. By a result of Ershov [18] there are index sets $I_{j}$ and filters $\mathscr{Q}_{j}$ with $X_{j}^{*} \equiv P\left(I_{j}\right) / \mathscr{Q}_{j}, 1 \leqslant j \leqslant m$. Without loss of generality we can assume the $I_{j}$ are pairwise disjoint; let $I=I_{1} \cup \cdots \cup I_{m}$. Define $\mathscr{D}$ to be the filter on $P(I)$ generated by $\left\{J_{1} \cup \cdots \cup J_{m} \mid J_{k} \in \mathscr{D}_{k}, 1 \leqslant k \leqslant m\right\}$. Then $P(I) / \mathscr{D} \cong \Pi_{1 \leqslant j \leqslant m} P\left(I_{j}\right) / \mathscr{D}_{j}$, and since $X(A)^{*} \cong I_{1 \leqslant j \leqslant m} X_{j}^{*}$, one has from Corollary 4.2 that the two first-order structures (Boolean algebras with $m$ distinguished points) $\left\langle X(A)^{*} ; X_{1}, \ldots, X_{m}\right\rangle$ and $\langle P(I) / \mathscr{D}$; $\left.I_{1} / \mathscr{D}, \ldots, I_{m} / \mathscr{D}\right\rangle$ are elementarily equivalent. Choose $A_{i} \in \Re$, for $i \in I_{j}$, such that $A_{i} \vDash \Theta_{j}, \quad 1 \leqslant j \leqslant m$. Then, since $X(A)^{*} \vDash \neg \Phi^{\prime}\left(X_{1}, \ldots, X_{m}\right.$, $\varnothing, \ldots, \varnothing)$, we also have

$$
P(I) / \mathscr{D} \vDash \neg \Phi^{\prime}\left(I_{1} / \mathcal{D}, \ldots, I_{m} / \mathscr{D}, 0, \ldots, 0\right),
$$

and hence $I_{i \in I} A_{i} / \mathscr{D} \vDash \neg \Phi$. 
(f) Suppose $A$ and $X^{*}$ satisfy the hypotheses of part (f) and $A[X]^{*}$ is not finite. To show $\operatorname{Th}\left(A[X]^{*}\right)$ is $\aleph_{0}$-categorical it suffices, by (d), to consider the case where $X^{*}$ is finite, or $X^{*}$ is denumerable with $\operatorname{Th}\left(X^{*}\right) \boldsymbol{\aleph}_{0}$-categorical. For any structure $B$ and elements $b_{1}, \ldots, b_{n} \in B$ the $n$-type of $b_{1}, \ldots, b_{n}$ in $B$ is $\left\{\Psi\left(u_{1}, \ldots, u_{n}\right) \mid B \vDash \Psi\left(b_{1}, \ldots, b_{n}\right)\right\}$. By a theorem of Ryll-Nardzewski (see [10]) a countable complete theory $T$ with no finite models is $\aleph_{0}$-categorical iff there are only finitely many (principal) $n$-types consistent with $T$ for each $n<\omega$. From our assumptions $\operatorname{Th}\left(A[X]^{*}\right)$ is complete and has no finite models. Fixing an $n<\omega$ suppose there are $l n$-types $\tau_{A}^{1}, \ldots, \tau_{A}^{l}$ realized in $A$ and $k l$-types $\tau_{X^{*}}^{1}, \ldots, \tau_{X^{*}}^{k}$ realized in $X^{*}$. If $f_{1}, \ldots, f_{n} \in A[X]^{*}$ let $\sigma(\vec{f})=$ $\{i\}$ if $X^{*} \vDash \tau_{X^{*}}^{i}\left(\left[\tau_{A}^{1}(\vec{f})\right], \ldots,\left[\tau_{A}^{l}(\vec{f}]\right)\right.$. The following claim is essentially due to Baldwin and Lachlan [3]: for $\vec{f}, \vec{g} \in A[X]^{*}$, if $\sigma(\vec{f})=\sigma(\vec{g})$ then $\vec{f}$ and $\vec{g}$ realize the same type in $A[X]^{*}$. To see this suppose $\sigma(\vec{f})=\sigma(\vec{g})=\{i\}$. Since $\operatorname{Th}\left(X^{*}\right)$ is categorical in the cardinality of $X^{*}$ it is straightforward to show that there are homeomorphisms $\lambda_{j}:\left[\tau_{A}^{j}(\vec{f})\right] \rightarrow\left[\tau_{A}^{j}(\vec{g})\right], 1 \leqslant j \leqslant l$, and these induce an isomorphism $\alpha: A[X]^{*} \rightarrow A[X]^{*}$ such that $\alpha(\vec{f})(x)$ has the same $n$-type in $A$ as $\bar{g}(x)$. But then from Theorem $4.1 \alpha(\vec{f})$ and $\vec{g}$ realize the same type in $A[X]^{*}$ as $(A ; \alpha(\vec{f})(x)) \equiv(A ; \vec{g}(x))$ for $x \in X^{*}$. This proves the claim, and hence part (f) via the above-mentioned characterization of $\boldsymbol{\aleph}_{0}$-categorical theories.

Remarks. (A) If $T h(\Re)$ is decidable then $T h\left(\Gamma^{e}(\Re)\right)$ is decidable as Tarski [35] has shown the theory of Boolean algebras is decidable.

(B) For any $\Omega, \operatorname{Th}\left(\Gamma^{e}(\Omega)\right)=\operatorname{Th}\left(\mathbf{P}_{R}(\Omega)\right)$. It would be interesting to know whether each member of $\Gamma^{e}(\Re)$ is elementarily equivalent to a member of $\mathbf{P}_{R}(\Omega)$, and vice-versa.

(C) For any $A$ and $T$,

$$
\operatorname{Th}\left(\Gamma_{T}^{e}(A)\right)=\operatorname{Th}\left(\left\{A^{I} / \mathscr{D} \mid P(I) / \mathscr{D} \vDash T\right\}\right) .
$$

Hence for any $B \in \Gamma^{e} \mathbf{S}^{\left({ }^{<}\right)} \mathbf{P}_{U}(A)$ there is an $I$ and $\mathscr{D}$ such that $B \equiv A^{I} / \mathscr{D}$; and thus the same holds for any $B \in \hat{\mathbf{P}}_{L R}(A)$ (Waszkiewicz and Weglorz [38]).

(D) A direct application of Theorem 4.1 and Corollary 4.2 shows that $A^{I} / \mathscr{D} \equiv A[P(I) / \mathscr{D}]^{*}$, for any $I$ and $\mathscr{D}$. Hence, given a countable language, if $A$ is finite or $\operatorname{Th}(A)$ is $\aleph_{0}$-categorical and if $P(I) / \mathscr{D}$ is finite or $\operatorname{Th}(P(I) / \mathscr{D})$ is $\aleph_{0}$-categorical then by (f) it follows that $\operatorname{Th}\left(A^{I} / \mathscr{D}\right)$ is $\aleph_{0}$-categorical if $A^{I} / \mathscr{D}$ is not finite (Waszkiewicz and Weglorz [39]).

5. An Ershov translation for filtered Boolean powers. Ershov [19] discovered that when working with bounded Boolean powers of a finite structure the Feferman and Vaught translation could be replaced by an intuitively clearer and more direct technique. In this section we will give a generalization of this which will be used for the decidability result in $\$ 6$. 
Throughout this section we will let $n$ be an arbitrary but fixed nonnegative integer. $\mathfrak{B} \mathfrak{A}^{(n)}$ denotes the class of Boolean algebras expanded by $n$ unary predicates $F_{1}, \ldots, F_{n}$ such that $\mathfrak{B O}^{(n)}$ satisfies " $F_{i}$ is a filter", $1 \leqslant i \leqslant n$, and $\mathfrak{X}^{(n)}$ denotes the class of sequences $\left\langle X_{0}, \ldots, X_{n}\right\rangle$ where $X_{0}$ is a Boolean space and $X_{1}, \ldots, X_{n}$ are closed subsets of $X_{0}$. We will conveniently abbreviate $\left\langle X_{0}, \ldots, X_{n}\right\rangle$ by $\bar{X}$, and $\bar{X}^{*}$ is the member of $\mathfrak{B O}^{(n)}$ corresponding to $\vec{X}$, i.e. $X_{0}^{*}$ with the predicates $F_{i}=\left\{Y \in X_{0}^{*} \mid X_{i} \subseteq Y\right\}$.

If $A$ is a structure let $\tilde{A}$ be an expansion of $A$ by $n$ unary predicates $A_{1}, \ldots, A_{n}$ such that each is a substructure of $A$. Then for $\vec{X} \in \mathfrak{X}^{(n)}$ let $\tilde{A}[\vec{X}]^{*}$ be the substructure of $A\left[X_{0}\right]^{*}$ whose universe is given by $\{f \in$ $\left.A\left[X_{0}\right]^{*} \mid f\left(X_{i}\right) \subseteq A_{i}, 1 \leqslant i \leqslant n\right\}$. This is our description of a typical filtered Boolean power (the construction is due to Arens and Kaplansky [1]). It is a simple exercise to show that $\tilde{A}[\vec{X}]^{*} \in \Gamma^{a} \mathbf{S}(\{A\})$. (Using sheaf-theoretic terminology $\tilde{A}[\vec{X}]^{*}$ is a structure of "global sections of a subsheaf of a constant sheaf over a Boolean space".) If $n=0$ then $\tilde{A}[\vec{X}]^{*}$ becomes $A\left[X_{0}\right]^{*}$, a bounded Boolean power.

From now on let $A$ be a particular finite structure as described above, say $A=\left\{a_{1}, \ldots, a_{\tilde{k}}\right\}$. If $\mathbf{f}$ is a fundamental operation of arity $m$ and $f_{1}, \ldots, f_{m+1} \in \tilde{A}[\vec{X}]^{*}$ then

$$
\begin{aligned}
\mathbf{f}\left(f_{1}, \ldots, f_{m}\right)=f_{m+1} \quad \text { iff } f_{1}^{-1}\left(a_{i_{1}}\right) & \cap \ldots \cap f_{m}^{-1}\left(a_{i_{m}}\right) \subseteq f_{m+1}^{-1}\left(a_{i_{m+1}}\right) \\
\text { whenever } A & \vDash \mathbf{f}\left(a_{i_{1}}, \ldots, a_{i_{m}}\right) \vDash a_{i_{m+1}} ;
\end{aligned}
$$

and if $\mathbf{r}$ is a fundamental $m$-ary relation of $A$ then $\mathbf{r}\left(f_{1}, \ldots, f_{m}\right)$ holds

$$
\operatorname{iff}\left[\underset{A \neq \mathbf{r}\left(a_{i}, \ldots, a_{i_{m}}\right)}{\bigcup} f_{1}^{-1}\left(a_{i_{1}}\right) \cap \cdots \cap f_{n}^{-1}\left(a_{i_{n}}\right)\right]=X_{0} .
$$

Also it is easy to see that for $f \in A^{X_{0}}$ we have

$$
\begin{array}{r}
f \in \tilde{A}[\vec{X}]^{*} \text { iff } f^{-1}\left(a_{1}\right), \ldots, f^{-1}\left(a_{k}\right) \in X_{0}^{*} \text { and } X_{i} \subseteq \bigcup_{a \in A_{i}} f^{-1}(a), \\
1 \leqslant i \leqslant n .
\end{array}
$$

Because of the finitary nature of (1)-(3) we will be able to show that:

TheOrem 5.1. For each formula $\Phi\left(u_{1}, \ldots, u_{l}\right)$ in the language of $A$ we can effectively find a formula $\hat{\Phi}\left(z_{11}, \ldots, z_{1 k} ; \ldots ; z_{l \underline{l}}, \ldots, z_{l k}\right)$ in the language of $\mathfrak{B} \mathfrak{A}^{(n)}$ such that for $\vec{X} \in \mathfrak{X}^{(n)}$ and $f_{1}, \ldots, f_{l}$ in $\tilde{A}[\bar{X}]^{*}$,

$$
\begin{aligned}
\tilde{A}[\vec{X}]^{*} \vDash & \Phi\left(f_{1}, \ldots, f_{l}\right) \\
& \text { iff } \bar{X}^{*} \vDash \hat{\Phi}\left(f_{1}^{-1}\left(a_{1}\right), \ldots, f_{1}^{-1}\left(a_{k}\right) ; \ldots ; f_{l}^{-1}\left(a_{1}\right), \ldots, f_{l}^{-1}\left(a_{k}\right)\right) .
\end{aligned}
$$


Proof. First we put $\Phi\left(u_{1}, \ldots, u_{l}\right)$ in a form $\Phi_{0}\left(u_{1}, \ldots, u_{l}\right)$, using only the two logical symbols Sheffer stroke $\mid$ and the existential quantifier, such that all atomic formulas are of the form $\mathrm{f}\left(u_{1}, \ldots, u_{m}\right)=u_{m+1}$, where $\mathrm{f}$ is a fundamental operation, or $\mathbf{r}\left(u_{1}, \ldots, u_{m}\right)$, where $\mathbf{r}$ is a fundamental relation, and $u_{1}, \ldots, u_{m+1}$ are variables. Now we give a recursive procedure to define $\hat{\Phi}_{0}$ and this will be $\hat{\Phi}$.

(i) If $\Phi_{0}$ is atomic: for $\mathbf{f}\left(u_{1}, \ldots, u_{m}\right)=u_{m+1}$ let $\hat{\Phi}_{0}$ be

$$
\left(\underset{A \vDash \mathbf{f}\left(a_{i,}, \ldots, a_{i_{m}}\right)=a_{i_{m+1}}}{\&}\left[z_{1 i_{1}} \wedge \cdots \wedge z_{m i_{m}} \leqslant z_{m+1, i_{m+1}}\right]\right) ;
$$

for $\mathbf{r}\left(u_{1}, \ldots, u_{m}\right)$ let $\hat{\Phi}_{0}$ be

$$
\left(\underset{A=\mathbf{r}\left(a_{i}, \ldots, a_{i_{m}}\right)}{\bigvee} z_{1 i_{1}} \wedge \cdots \wedge z_{m i_{m}}\right)=1 .
$$

(ii) Given $\hat{\Phi}_{1}$ and $\hat{\Phi}_{2}$ let $\widehat{\Phi_{1} \mid \Phi_{2}}$ be $\hat{\Phi}_{1} \mid \hat{\Phi}_{2}$.

(iii) To handle the existential quantifier first define $\operatorname{Adm}\left(z_{1}, \ldots, z_{k}\right)$ to be:

$$
\begin{aligned}
\left(\underset{1<i<j<k}{\&} z_{i} \wedge z_{j}=0\right) \&\left(z_{1} \vee \cdots\right. & \left.\vee z_{k}=1\right) \\
& \& \underset{1<i<k}{\&}\left(\bigvee\left\{z_{j} \mid a_{j} \in A_{i}\right\} \in F_{i}\right) .
\end{aligned}
$$

Then $\widehat{\exists u_{1} \Phi}$ is $\exists z_{11} \ldots \exists z_{1 k}\left[\operatorname{Adm}\left(z_{11}, \ldots, z_{1 k}\right) \& \hat{\Phi}\right]$.

Next we proceed by induction to show that this definition of $\hat{\Phi}$ suffices. For the atomic case just use (1) and (2) above. Verification for the case (ii) is straightforward. For (iii) suppose $\tilde{A}[\vec{X}]^{*} \vDash \exists u_{1} \Phi\left(u_{1}, f_{2}, \ldots, f_{l}\right)$. Then, choosing $f_{1}$ such that $\tilde{A}[\bar{X}]^{*}=\Phi\left(f_{1}, \ldots, f_{l}\right)$ we have $\operatorname{Adm}\left(f_{1}^{-1}\left(a_{1}\right), \ldots, f_{1}^{-1}\left(a_{k}\right)\right)$ by $(3)$, and $\hat{\Phi}\left(f_{1}^{-1}\left(a_{1}\right), \ldots, f_{1}^{-1}\left(a_{k}\right) ; \ldots ; f_{l}^{-1}\left(a_{1}\right), \ldots, f_{l}^{-1}\left(a_{k}\right)\right)$ holds by the induction hypothesis. Conversely, if $X^{*}$ satisfies

$$
\begin{array}{r}
\exists z_{11} \ldots \exists z_{1 k}\left[\operatorname{Adm}\left(z_{11}, \ldots, z_{1 k}\right) \& \hat{\Phi}\left(z_{11}, \ldots, z_{1 k} ; f_{2}^{-1}\left(a_{1}\right), \ldots, f_{2}^{-1}\left(a_{k}\right)\right) ;\right. \\
\left.\ldots ; f_{l}^{-1}\left(a_{1}\right), \ldots, f_{l}^{-1}\left(a_{k}\right)\right]
\end{array}
$$

then letting $f_{1} \in A^{X_{0}}$ be defined by $f_{1}(x) \vDash a_{i}$ if $x \in Z_{1 i}$, where $Z_{11}, \ldots, Z_{1 k}$ witness the $z_{11}, \ldots, z_{1 k}$, it is clear that $f_{1} \in \tilde{A}[\vec{X}]^{*}$ and $X^{*} \vDash \hat{\Phi}\left(f_{1}^{-1}\left(a_{1}\right)\right.$, $\left.\ldots, f_{l}^{-1}\left(a_{k}\right)\right)$ as $f_{1}^{-1}\left(a_{i}\right)=Z_{1 i}$, hence $\tilde{A}[\vec{X}]^{*} \vDash \Phi\left(f_{1}, \ldots, f_{l}\right)$, so it follows that $\tilde{A}[\vec{X}]^{*} \vDash \exists u_{1} \Phi\left(u_{1}, f_{2}, \ldots, f_{l}\right)$. This finishes the proof.

An immediate application of Theorem 5.1 is:

Corollary 5.2. Let $\mathfrak{Y}$ be a subclass of $\mathfrak{X}^{(n)}$ such that the theory of $\left\{\overrightarrow{X^{*}} \mid \vec{X} \in \mathfrak{Y}\right\}$ is decidable. Then the theory of $\left\{\tilde{A}[\vec{X}]^{*} \mid \vec{X} \in \mathfrak{Y}\right\}$ is decidable. In particular if $\Phi$ is a sentence in the language of $\mathfrak{B Q}^{(n)}$ then the theory of $\left\{\tilde{A}[\vec{X}]^{*} \mid \vec{X}^{*}\right.$ is countable and $\left.\vec{X}^{*} \vDash \Phi\right\}$ is decidable. 
Proof. The first assertion is clear. The second follows from the decidability of the theory of countable Boolean algebras with quantification over filters (Rabin [31]).

6. A decidability result for $\Gamma_{\omega}^{a}$. For $\Re$ a class of structures let $\Gamma_{\omega}^{a}(\Re)$ be the class of $A$ in $\Gamma^{a}(\Re)$ such that $X(A)^{*}$ is a countable Boolean algebra.

THEOREM 6.1. Let $\Re$ be a finite collection of finite structures with $L$ a finite language. Then $\Gamma_{\omega}^{a}(\Re)$ has a decidable theory.

The core of the proof is to construct a finite structure $H$ and an expansion $\tilde{H}$ (say by $H_{1}, \ldots, H_{n}$ ), and to find a sentence $\Phi$ in the language of $\mathfrak{B O}^{(n)}$ such that, up to isomorphism, $\Gamma_{\omega}^{a}(\Re)$ and $\left\{\tilde{H}[\vec{X}]^{*} \mid \vec{X} \in \mathfrak{X}_{\omega}^{(n)}\right.$ and $\left.\vec{X}^{*} \models \Phi\right\}$ are the same classes, where $\mathfrak{X}_{\omega}^{(n)}$ is the class of $\bar{X} \in \mathfrak{X}^{(n)}$ such that $X_{0}^{*}$ is countable. ${ }^{7}$ Then, by Corollary 5.2, the theory of $\Gamma_{\omega}^{a}(\Re)$ is decidable.

So let $\Re=\left\{A_{1}, \ldots, A_{m}\right\}$, and let $\leqslant$ be the embeddability partial-ordering on $\Re$, i.e. $A_{i} \leqslant A_{j}$ iff there exists an embedding of $A_{i}$ into $A_{j}$. Since the collection of finite $L$-structures has the joint embedding property and the amalgamation property we can find finite structures $P_{1}, \ldots, P_{m}$ and $H$ such that

(a) $A_{i}$ is a substructure of $P_{i}, 1 \leqslant i \leqslant m$,

(b) if $A_{j} \leqslant A_{i}$ then, given an embedding $\alpha: A_{j} \rightarrow P_{i}$ there is an embedding $\beta: P_{i} \rightarrow P_{j}$ such that $\beta \circ \alpha$ is the identity map on $A_{j}$, and

(c) each $P_{i}$ can be embedded into $H$.

For the rest of this proof let $P_{1}, \ldots, P_{m}$ and $H$ be fixed finite $L$-structures satisfying (a)-(c), and then let $H_{1}, \ldots, H_{n}$ be a listing of all the substructures of $H$ which are isomorphic to a member of $\Re$. Define $\tilde{H}$ to be the expansion of $H$ by these substructures (as in $\S 5$ ).

Now we are ready to define $\Phi$ as the sentence:

$$
\forall x\left[\left(\underset{1<i<n}{\&} x \in F_{i}\right) \rightarrow x=1\right] \& \underset{1<i, j<n}{\&}\left[F_{i} \vee F_{j}=\bigcap_{H_{k} \subseteq H_{i} \cap H_{j}} F_{k}\right] .
$$

First we will show that if $\vec{X}$ is in $\mathfrak{X}_{\omega}^{(n)}$ and $\vec{X}^{*} \vDash \Phi$, then $\tilde{H}[\vec{X}]^{*} \in \Gamma_{\omega}^{a}(\mathbf{I} \Re)$. $X^{*} \vDash \Phi$ means $X_{1} \cup \cdots \cup X_{n}=X_{0}$ and, for $1 \leqslant i, j \leqslant n, X_{i} \cap X_{j}=$ $\cup_{H_{k} \subseteq H_{i} \cap H_{i}} X_{k}$. Consequently given $x \in X_{0}$ there is a smallest member (under $\subseteq$ ), say $H_{i_{x}}$, of $H_{1}, \ldots, H_{n}$ such that $x \in X_{i_{x}}$; and hence if $x \in X_{j}$ then $H_{i_{x}} \subseteq H_{j}$. As the $X_{i}$ 's are closed subsets of $X_{0}$ it follows that there is a clopen neighborhood $N_{x}$ of $x$ such that for $1 \leqslant j \leqslant n, X_{j} \cap N_{x} \neq \varnothing$ implies $x \in X_{j}$, hence $H_{i_{x}} \subseteq H_{j}$. Applying compactness we can conclude that there are

${ }^{7}$ Comer [13] developed this proof technique for monadic algebras. However, his definition of $H$ was considerably complicated by requiring that it also be a monadic algebra. The simplification we are using is due to Werner. Also it should be noted that Comer used a version of the Feferman and Vaught translation rather than our $\$ 5$. 
finitely many disjoint clopen subsets $N_{0}, \ldots, N_{l}$ of $X_{0}$ which cover $X_{0}$, and corresponding predicates $H_{i_{0}}, \ldots, H_{i_{L}}$ of $\tilde{H}$, such that if $f \in H\left[X_{0}\right]^{*}$ and $f\left(N_{j}\right) \subseteq H_{i}, 0 \leqslant j \leqslant l$, then $f \in \tilde{H}[\vec{X}]^{*}$. Thus $\tilde{H}[\vec{X}]^{*}$ is nonempty (if $H$ and $X$ are nonempty); and $\left(\tilde{H}[\vec{X}]^{*}\right)_{x}=H_{i_{x}}$ as one sees by making sure $N_{x}$ appears in the list $N_{0}, \ldots, N_{l}$. This proves that $\tilde{H}[\vec{X}]^{*}$ is in $\Gamma_{\omega}^{a}(I \Omega)$.

Next we show that every $A \in \Gamma_{\omega}^{a}(\Re)$ has the desired representation by a filtered Boolean power. The following lemma contains the key observation.

Lemma 6.2. Let $A \in \Gamma_{\omega}^{a}(\Re)$. For each $x \in X(A)$ there is an $N \in X(A)^{*}$, with $x \in N$, and there are embeddings $\sigma_{y}: A_{y} \rightarrow H$, for each $y$ in $N$, such that given $f \in A$ and $h \in H,\left\{y \in N \mid \sigma_{y}(f(y))=h\right\}$ is clopen in $X(A)$.

Let $\lambda_{x}: A_{i} \rightarrow A_{x}$ be an isomorphism for suitable $A_{i} \in \Re$. Actually we will show, by downward induction on the $\leqslant$-rank of $A_{i}$, that for $y \in N$ there are embeddings $\sigma_{y}: A_{y} \rightarrow P_{i}$ with the desired property. This will suffice as $P_{i}$ can be embedded in $H$.

Case a. $A_{i}$ is $\leqslant$-maximal. Choose $f_{1}, \ldots, f_{m} \in A$ such that $A_{x}=$ $\left\{f_{1}(x), \ldots, f_{m}(x)\right\}$, and let $D_{x}\left(u_{1}, \ldots, u_{m}\right)$ be the set of atomic and negated atomic formulas $\Phi(\vec{u})$ such that $A_{x} \vDash \Phi(\vec{f}(x))$. Define $N$ to be the clopen set $\left[\& D_{x}\left(f_{1}, \ldots, f_{m}\right)\right]$. Then for $y \in N$ the mapping $\lambda_{x y}: A_{x} \rightarrow A_{y}$ defined by $\lambda_{x y}\left(f_{j}(x)\right)=f_{j}(y), 1 \leqslant j \leqslant m$, is an isomorphism. Letting $\sigma_{y}=\lambda_{x}^{-1} \circ \lambda_{x y}^{-1}$ we have an embedding of $A_{y}$ into $A_{i}$, and hence into $P_{i}$. Then given $h \in P_{i}$ and $f \in A$ we have $\left\{y \in N \mid \sigma_{y}(f(y))=h\right\}=N \cap\left[f=f_{j}\right]$ if $\lambda_{x}(h)=f_{j}(x)$.

Case b. There is a clopen subset $M$ such that $x \in M$ and $A_{x} \cong A_{y}$ for $y \in M$. Then we can argue as in Case (a).

Case c. Suppose Case (b) does not hold at $x$. Choose $f_{1}, \ldots, f_{m} \in A$ such that $A_{x}=\left\{f_{1}(x), \ldots, f_{m}(x)\right\}$, and define $D_{x}$ and then $N$ and the $\lambda_{x y}$ as in Case (a). Then for $y \in N$ we have $\lambda_{x y}: A_{x} \rightarrow A_{y}$ is an embedding. If for a given $y \in N$ the map $\lambda_{x y}$ is not onto then, looking at a $D_{y}$ one sees that there is a neighborhood of $y$ for which $\lambda_{x z}$ is not onto for $z$ in this neighborhood of $y$. Letting $Y$ be the subset of $N$ for which $\lambda_{x y}$ is onto, $y \in Y$, we see that $Y$ must be closed. For $y \in Y$ define $\sigma_{y}$ to be $\lambda_{x}^{-1} \circ \lambda_{x y}^{-1}$. Now $N-Y$ is open and $X(A)^{*}$ is a countable Boolean algebra, hence $N-Y$ is a disjoint union of countably many clopen subsets. For $y \in N-Y$ the $\leqslant-$ rank of $A_{y}$ is higher than that of $A_{x}$, so by induction we have, for each $y \in N-Y$, the conclusions of the lemma. Hence it follows that there is a countable sequence of pairwise disjoint clopen subsets $N_{k}, k<\omega$, with $\cup_{k<\omega} N_{k}=N-Y$, and for each $N_{k}$ a family of maps $\hat{\sigma}_{y}, y \in N_{k}$, such that the conclusion of the lemma holds, except that $H$ is replaced by an appropriate $P_{n_{k}}$, with $A_{i}<A_{n_{k}}$. Let $\varepsilon_{i}: A_{i} \rightarrow P_{i}$ be the inclusion map. For $y \in N_{k}$ choose an embedding $\mu_{y}$ such that the following diagram commutes: 


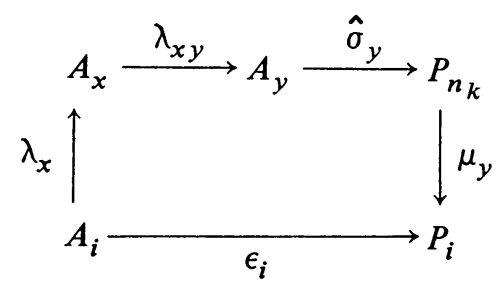

Then define $\sigma_{y}$ to be $\mu_{y} \hat{\sigma}_{y}$. Thus, for $y \in N, \sigma_{y}\left(f_{j}(y)\right)=\sigma_{x}\left(f_{j}(x)\right)$.

To finish the proof of the lemma suppose $f \in A$ and $h \in P_{i}$. If $h \in A_{i}$ then $\left\{y \in N \mid \sigma_{y}(f(y))=h\right\}=\left[f=f_{j}\right] \cap N$ if $h=f_{j}(x)$; and if $h \notin A_{i}$ then

$$
\begin{aligned}
\left\{y \in N \mid \sigma_{y}(f(y))=h\right\} & =\bigcup_{k<\omega}\left\{y \in N_{k} \mid \mu_{y} \hat{\sigma}_{y}(f(y))=h\right\} \\
& =\bigcup_{k<\omega}\left\{y \in N_{k} \mid \hat{\sigma}_{y}(f(y))=\mu_{y}^{-1}(h)\right\},
\end{aligned}
$$

an open set. Now the open sets $\left\{y \in N \mid \sigma_{y}(f(y))=h\right\}, h \in P_{i}$, form a finite disjoint cover of $N$, hence they are actually all clopen.

LEMma 6.3. For $X_{0}^{*}$ a countable Boolean algebra and $B$ a substructure of $H\left[X_{0}\right]^{*}$ let $B(x)=\{f(x) \mid f \in B\}$, for $x \in X_{0}$. Then there is an $\vec{X} \in \mathfrak{X}_{\omega}^{(n)}$ such that $\bar{X}^{*}=\Phi$ and $B=\tilde{H}[\bar{X}]^{*}$ iff

(i) $B(x) \in\left\{H_{1}, \ldots, H_{n}\right\}$, for $x \in X_{0}$, and

(ii) $B$ has the Patchwork Property (3).

Proof. First note that any substructure of $H\left[X_{0}\right]^{*}$ satisfies $\left(2^{a}\right)$.

$\Leftrightarrow)$ In the discussion just before the statement of Lemma 6.2 we showed that if $\vec{X}$ is in $\mathfrak{X}_{\omega}^{(n)}$ and $\vec{X}^{*} \vDash \Phi$ then $\left(\tilde{H}[\vec{X}]^{*}\right)_{x}$ is in $\left\{H_{1}, \ldots, H_{n}\right\}$, for $x \in X_{0}$. To see that $\tilde{H}[\vec{X}]^{*}$ satisfies (3) one merely checks the definition of a filtered Boolean power.

$(\Leftarrow)$ As the elements of $H\left[X_{0}\right]^{*}$ are locally constant and $H$ is finite we see that for any $j, 1 \leqslant j \leqslant n$, the set $\left\{x \in X_{0} \mid H_{j} \subseteq B(x)\right\}$ is an open subset of $X_{0}$. Define $\vec{X}$ by letting $X_{0}$ be as above and letting $X_{j}=\left\{x \in X_{0} \mid B(x) \subseteq\right.$ $\left.H_{j}\right\}, 1 \leqslant j \leqslant n$. An easy argument shows that $X_{j}=\cap_{H_{k} \subseteq H_{j}}\left\{x \in X_{0} \mid H_{k} \&\right.$ $B(x)\}$, hence each $X_{j}$ is indeed closed. Then, using (i), it is also easy to see that $\vec{X}^{*} \vDash \Phi$, and obviously $B \subseteq \tilde{H}[\vec{X}]^{*}$ as $x \in X_{j}$ and $f \in B$ imply $f(x) \in$ $H_{\dot{L}}$. Combining (i) with the discussion preceding Lemma 6.2 it follows that $\left(\tilde{H}[\vec{X}]^{*}\right)_{x}=B(x)$ for $x \in X_{0}$; but then (3) guarantees $B=\tilde{H}[\vec{X}]^{*}$.

Now to finish the proof of Theorem 6.1 let us look again at $A \in \Gamma_{\omega}^{a}(\Re)$ and use Lemma 6.2 and compactness to find finitely many pairwise disjoint clopen sets $N_{1}, \ldots, N_{k}$ which cover $X(A)$, and corresponding systems of homomorphisms $\sigma_{y}$ as described in Lemma 6.2. Define $\sigma: A \rightarrow H^{X(A)}$ by $\sigma(f)(x)=\sigma_{x}(f(x))$. Clearly $\sigma$ is an embedding, $\sigma(A)(x)$ is in $\left\{H_{1}, \ldots, H_{n}\right\}$ for $x \in X(A)$, and $\sigma(A)$ satisfies (3) since $A$ satisfies (3). For $f \in A$ and 
$h \in H$ we have

$$
\{y \in X(A) \mid \sigma(f)(y)=h\}=\bigcup_{1<j<k}\left\{y \in N_{k} \mid \sigma_{y}(f(y))=h\right\},
$$

a clopen subset of $X(A)$, hence $\sigma(A)$ is a substructure of $H[X(A)]^{*}$. Thus from Lemma $6.3, \sigma(A)=\tilde{H}[\vec{X}]^{*}$ where $X_{0}=X(A)$ and, for $1 \leqslant j \leqslant n$, $X_{j}=\left\{x \in X(A) \mid \sigma(A)(x) \subseteq H_{j}\right\}$.

Remark 1. For $A \in \Gamma^{a}(\Re)$ let us define a closed relation on $A$ to be any binary relation of the form $\left\{\langle f, g\rangle \in A^{2} \mid[f=g] \supseteq Y\right\}$, where $Y$ is a closed subset of $X(A)$. It is easy to extend the translation of $\$ 5$ to cover the closed relations, namely (with $U$ a variable for closed relations and $V$ a variable for filters):

$$
\begin{aligned}
& \text { if } \Phi_{0} \text { is of the form }\left\langle u_{1}, u_{2}\right\rangle \in U \text { then let } \hat{\Phi}_{0} \text { be }\left[\bigvee_{1 \leqslant i \leqslant k} z_{1 i} \wedge z_{2 i}\right] \\
& \in V \text {; and given } \hat{\Phi}_{0} \text { let } \exists U \Phi_{0} \text { be } \exists V \hat{\Phi}_{0} .
\end{aligned}
$$

Then Corollary 5.2 can be changed to assert: 'if the theory of $\left\{\vec{X}^{*} \mid \vec{X} \in \mathfrak{Y}\right\}$ with quantification over filters, is decidable, then the theory of $\left\{\tilde{A}[\vec{X}]^{*} \mid \vec{X} \in\right.$ $\mathfrak{Y}\}$ with quantification over closed relations is decidable'. Hence the conclusion of Theorem 6.1 can be strengthened to state: Then $\Gamma_{\omega}^{a}(\Omega)$ has a decidable theory with quantification over closed relations. We presented essentially this form in the special case $\Gamma_{\omega}^{a}(\mathbf{S}(\Re))$, where $\Re$ is a class of algebras, in [7] and in Werner [42], the emphasis in both cases being on discriminator varieties (see Remark 2)-in discriminator varieties the closed relations of $A$ are precisely the congruences of $A$.

REMARK 2. The key applications of Theorem 6.1 have been to classes $\Re$ such that $I \Gamma^{a}(\Re)$ is elementary, for then the theory of $\Gamma^{a}(\Re)$ is the same as that of $\Gamma_{\omega}^{a}(\Re)$, assuming the members of $\Re$ are finite. The interesting examples we know of are discriminator varieties, that is, equationally defined classes $\mathfrak{B}$ of algebras such that there is a term $t\left(u_{1}, u_{2}, u_{3}\right)$ with the class $\subseteq$ of subdirectly irreducible members of $\mathfrak{B}$ satisfying:

$$
t\left(u_{1}, u_{2}, u_{3}\right)=u_{4} \Leftrightarrow\left(u_{1}=u_{2} \& u_{3}=u_{4}\right) \bigvee\left(u_{1} \neq u_{2} \& u_{1}=u_{4}\right) .
$$

It is proved in Keimel and Werner [24], for the case $\subseteq$ is finite, and in general in Bulman-Fleming and Werner [5] that for such $\mathfrak{B}, \mathfrak{B}=\mathbf{I} \Gamma^{a}(\Im)$. (See $\S 9$ for our treatment of discriminator varieties.)

Shortly after Comer [13] announced the decidability of residually finite varieties of monadic algebras Quackenbush pointed out that these are discriminator varieties. Subsequently Werner modified Comer's proof in order to include all residually finite discriminator varieties (those for which $\subseteq$ is finite), and this applies to (add residually finite for (a)) varieties of:

(a) cylindric algebras,

(b) Post algebras, 
(c) Kukasiewicz algebras,

(d) relatively complemented distributive lattices, and

(e) commutative rings with unity satisfying $x^{m}=x$, for any given $m>1$.

For complete details on these and other examples see Werner [42]. Using the stronger form of Theorem 6.1 mentioned in Remark 1 we announced (in slightly different words) [8]:

the first-order theory, with quantification over congruences, of the countable algebras in a residually finite discriminator variety is decidable.

Surprisingly this covers most of the equationally defined classes which are known to have a decidable theory, the most important examples being:

(1) Boolean algebras (Tarski [35], 1949),

(2) relatively complemented distributive lattices (Ershov [18], 1964),

(3) $n$-valued Post algebras (Ershov [19], 1967),

(4) countable Boolean algebras with quantification over ideals (Rabin [31], 1969), ${ }^{8}$

(5) $\left(x^{m}=x\right)$-rings (Comer [12], 1974), and

(6) residually finite varieties of monadic algebras (Comer [13], 1975).

Notable exceptions to this list are:

(7) Abelian groups (Szmielew [34], 1954), and

(8) mono-unary algebras (Ehrenfeucht [16], 1959). ${ }^{9}$

Remark 3. Arens and Kaplansky [1] showed that if $R$ is a finite field (considered as a ring with unity) then every countable member of the variety generated by $R$ is a filtered Boolean power of $R$. (Comer used this to prove (5).) We can derive this result by looking at the proof of Theorem 6.1 and letting $H$ be $R$, the $P_{i}$ also equal $R$, and the $A_{j}$ the various subfields of $R$. This particularly nice representation of Arens and Kaplansky can be generalized to any $A$ which is infraprimal (see Werner [42]).

REMARK 4. Looking at the list of varieties with decidable theories given in Remark 2 we were struck by the fact that they are all residually small, i.e. they do not have arbitrarily large subdirectly irreducible members. However McKenzie soon proved that if one takes the variety generated by $\langle\omega, t\rangle, t$ being the ternary discriminator on $\omega$, then it is not residually small, but it does have a decidable theory. We will present further examples in $\$ 9$.

REMARK 5. Rubin [32] has proved that the theory of monadic algebras is not decidable. Let $\Re$ be the class of Boolean algebras with an additional unary

\footnotetext{
${ }^{8}$ Of course this is the result we use to prove Theorem 6.1.

${ }^{9}$ As is well known, (1), (2) and (7) were originally proved by quantifier elimination (introduced by Skolem in 1919). Ershov proved (3) by combining (1) with Foster's Boolean power representation of Post-algebras. Ehrenfeucht used semi-models.
} 
operation $c$ satisfying $c(u)=1$ if $u \neq 0$, and $c(0)=0$. Then the countable monadic algebras are just the countable members of $\mathbf{I} \Gamma^{a}(\Re)$, hence $\Gamma_{\omega}^{a}$ does not preserve decidability (in contrast to $\Gamma^{e}$ ).

7. The operators $\Gamma_{U}^{e}, \Gamma_{0}^{e}, \Gamma_{0}^{a}$. In this section we define a generalization of the ultraproduct construction and use it to strengthen Theorem 2.1(d). This will be used in the study of model companions in the next three sections.

Suppose $A_{i} \in \Gamma^{e}(\Re)$, for $i \in I$, and $\mathcal{U}$ is an ultrafilter in $\prod_{i \in I} X\left(A_{i}\right)^{*}$. Define an equivalence relation $\sim_{\mathcal{Q}}$ on $\Pi_{i \in I} A_{i}$ by $f \sim_{\mathcal{Q}} g$ iff $\{\langle i,[f(i)=g(i)]\rangle \mid i \in I\} \in \mathcal{Q}$, and for $f \in \prod_{i \in I} A_{i}$ let the equivalence class of $f$ be denoted by $f / \mathcal{U}$. Then the structure $\Pi_{i \in I} A_{i} / \mathcal{U}$ is defined by letting its universe be $\left\{f / \mathcal{U} \mid f \in \prod_{i \in I} A_{i}\right\}$ and then, for an atomic formula $\Phi(\vec{u})$ and elements $\vec{f}$ in $\Pi_{i \in I} A_{i}$, we say $\Pi_{i \in I} A_{i} / \mathscr{U} \vDash \Phi(\bar{f} / \mathscr{U})$ iff $\{\langle i,[\Phi(\vec{f}(i))]\rangle \mid i \in I\} \in \mathcal{Q}$. The structure $\prod_{i \in I} A_{i} / \mathcal{U}$ is what the members of $\Gamma_{U}^{e}(\Omega)$ look like.

Lemma 7.1. Suppose $A_{i} \in \Gamma^{e}(\Re)$, for $i \in I$, and $\mathscr{U}$ is an ultrafilter in $\prod_{i \in I} X\left(A_{i}\right)^{*}$. Then, for any $L$-formula $\Phi\left(u_{1}, \ldots, u_{m}\right)$ and elements $f_{1}, \ldots, f_{m}$ $\in \prod_{i \in I} A_{i}$, we have $\prod_{i \in I} A_{i} / \mathcal{U} \vDash \Phi\left(f_{1} / \mathcal{U}, \ldots, f_{m} / \mathcal{U}\right)$ iff $\left\{\left\langle i,\left[\Phi\left(f_{1}(i), \ldots, f_{m}(i)\right)\right]\right\rangle \mid i \in I\right\} \in \mathcal{U}$.

Proof. We proceed by induction on the complexity of the formula $\Phi(\vec{u})$. The atomic case is just the definition of " $\models$ ". Next, for any $\Phi(\vec{u})$, $\mathcal{U}$ contains exactly one of $\{\langle i,[\Phi(\vec{f}(i))]\rangle \mid i \in I\}$ and $\{\langle i,[\neg \Phi(\vec{f}(i))]\rangle \mid i \in I\}$, and since $\mathcal{U}$ is closed under meet, the induction steps for $\neg$ and $\&$ are straightforward. So suppose we have proved the theorem for the formula $\Phi(u, \vec{u})$. If $\prod_{i \in I} A_{i} / \mathcal{Q} \vDash \exists u \Phi(u, \vec{f} / \mathcal{Q})$ then choose $f \in \prod_{i \in I} A_{i}$ such that $\prod_{i \in I} A_{i} /$ Q $\Phi(f / \mathcal{Q}, \vec{f} / \mathcal{Q})$. Then $\{\langle i,[\Phi(f(i), \vec{f}(i))]\rangle \mid i \in I\} \in \mathcal{Q}$ by assumption, hence $\{\langle i,[\exists u \Phi(u, \vec{f}(i))]\rangle \mid i \in I\} \in \mathcal{Q}$. Conversely, if $\{\langle i,[\exists u \Phi(u, \vec{f}(i))]\rangle \mid i \in I\} \in$ $\mathcal{Q}$ we can apply the Maximal Property to find $g_{i} \in A_{i}$ such that $[\exists u \Phi(u$, $\vec{f}(i))]=\left[\Phi\left(g_{i}, \vec{f}(i)\right)\right]$, for $i \in I$. Then choosing $f \in \prod_{i \in I} A_{i}$ defined by $f(i)=g_{i}$, $i \in I$, we have $\{\langle i,[\Phi(f(i), \vec{f}(i))]\rangle \mid i \in I\} \in \mathcal{Q}$, so by the induction hypothesis, $\Pi_{i \in I} A_{i} / \mathcal{U} \vDash \Phi(f / \mathcal{U}, \vec{f} / \mathcal{U})$, and hence it follows that $\prod_{i \in I} A_{i} / \mathcal{U}$ $\vDash \exists u \Phi(u, \vec{f} /$ थ $)$.

Lemma 7.2. (a) $\mathbf{P}_{R} \Gamma^{e} \leqslant \Gamma^{e} \Gamma_{U}^{e}$, and

(b) $\mathbf{P}_{U} \leqslant \Gamma_{U}^{e} \leqslant \mathbf{S}^{(<)} \mathbf{P}_{U}$.

Proof. (a) Suppose $A_{i} \in \Gamma^{e}(\Re)$ for $i \in I$ and that $\mathscr{D}$ is a filter on $P(I)$. Let $\lambda$ be the canonical map from $\operatorname{II}_{i \in I} X\left(A_{i}\right)^{*}$ onto $\prod_{i \in I} X\left(A_{i}\right)^{*} / \mathscr{D}$. Let $X=$

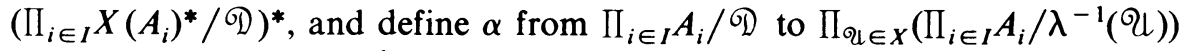
by $\alpha(f / \mathscr{D})(\mathscr{Q})=f / \lambda^{-1}(\mathcal{Q})$. Clearly $\alpha$ is a homomorphism, and the image of $\alpha$ is a subdirect product of the $\prod_{i \in I} A_{i} / \lambda^{-1}(\mathcal{Q})$ for $\mathcal{U} \in X$. If $\Phi(\vec{u})$ is any 
formula and $\vec{f} \in \prod_{i \in I} A_{i}$ then $\mathcal{Q} \in[\Phi(\alpha(\vec{f} / \mathscr{D}))]$ iff $\prod_{i \in I} A_{i} / \lambda^{-1}(\mathscr{Q})=$ $\Phi\left(\vec{f} / \lambda^{-1}(\mathcal{U})\right)$ iff $\{\langle i,[\Phi(\vec{f}(i))]\rangle \mid i \in I\} \in \lambda^{-1}(\mathcal{U})$. From this we can conclude that $\alpha$ is injective and the image of $\alpha$ satisfies $\left(2^{e}\right)$. For (3) let $f, g \in$ $\prod_{i \in I} A_{i}$ and let $N$ be a clopen subset of $X$. Then for some choice of $\left\{\left\langle i, J_{i}\right\rangle \mid i \in I\right\}$, where $J_{i} \in X\left(A_{i}\right)^{*}$, we have, for $\mathcal{Q} \in X$, $\mathscr{Q} \in N$ iff $\left\{\left\langle i, J_{i}\right\rangle \mid i \in I\right\} \in \lambda^{-1}(\mathscr{Q})$. Let $h$ be defined by $h(i)(x)=f(i)(x)$ if $i \in I$ and $x \in J_{i}$, and otherwise $h(i)(x)=g(i)(x)$. Then, for $\mathscr{U} \in N$, $\mathcal{Q} \in[\alpha(h / \mathscr{Q})=$ $\alpha(f / \mathscr{D})]$ since $\left\{\left\langle i, J_{i}\right\rangle \mid i \in I\right\} \leqslant\{\langle i,[h(i)=f(i)]\rangle \mid i \in I\}$, and similarly $\mathcal{Q}$ $\in X-N$ implies $\mathscr{Q} \in[\alpha(h / \mathscr{D})=\alpha(g / \mathscr{D})]$, hence $\alpha(h / \mathscr{D})=\alpha(f / \mathscr{D}) \uparrow_{N}$ $\cup \alpha(g / \mathscr{D}) \uparrow_{X-N}$.

(b) To see that $\mathbf{P}_{U} \leqslant \Gamma_{U}^{e}$ let $X$ be the one-element Boolean space, and make use of the obvious isomorphism between $\prod_{i \in I} A_{i}$ and $\prod_{i \in I} A_{i}[X]^{*}$ and the isomorphism between $P(I)$ and $\left(X^{*}\right)^{I}$. For the second inequality in (b) suppose $A_{i} \in \Gamma^{e}(\Re)$ for $i \in I$, say $A_{i}$ is a subdirect product of $A_{x}^{i}$ for $x \in X\left(A_{i}\right)$, and let $\mathcal{Q}$ be an ultrafilter in $\prod_{i \in I} X\left(A_{i}\right)^{*}$. Let $I^{\prime}=\{\langle i, x\rangle \mid i \in I$, $\left.x \in X\left(A_{i}\right)\right\}$. Then the mapping $\beta$ from $\Pi_{i \in I} A_{i}$ into $\Pi_{\langle i, x\rangle \in I^{\prime}} A_{x}^{i}$ defined by $\beta(f)(\langle i, x\rangle)=f(i)(x)$ is an embedding. Let $\mho \int$ be an ultrafilter on $P\left(I^{\prime}\right)$ extending $\left\{\left\{\langle i, x\rangle \in I^{\prime} \mid x \in U(i)\right\} \mid U \in \mathcal{Q}\right\}$, and let $\beta\left(\prod_{i \in I} A_{i}\right) / \mathscr{Q}$ be the substructure of $\prod_{\langle i, x\rangle \in I^{\prime}} A_{x}^{i} / \mathscr{W}$ with universe $\left\{\beta(f) / \mathscr{W} \mid f \in \prod_{i \in I} A_{i}\right\}$. Our definition ensures that for $\Phi(\vec{u})$ an atomic $L$-formula and $\vec{f}$ in $\prod_{i \in I} A_{i}$, $\beta\left(\prod_{i \in I} A_{i}\right) / \mathscr{W} \vDash \Phi(\beta(\vec{f}) / \mathscr{W})$ iff $\prod_{i \in I} A_{i} / \mathcal{U}=\Phi(\vec{f} / \mathcal{Q})$, and hence the mapping $\gamma: \prod_{i \in I} A_{i} / \mathcal{U} \rightarrow \beta\left(\Pi_{i \in I} A_{i}\right) / \mathcal{Q}$ defined by $\gamma(f / \mathcal{Q})=\beta(f) / \mho$ is an isomorphism. To see that $\beta\left(\Pi_{i \in I} A_{i}\right) / \mathscr{W}$ is an elementary substructure of $\Pi_{\langle i, x\rangle \in I^{\prime}} A_{x}^{i} / \mathscr{Q}$ let $\Phi(\vec{u})$ be any $L$-formula and suppose $\vec{f} \in \Pi_{i \in I} A_{i}$. Then $\beta\left(\prod_{i \in I} A_{i}\right) / \mathscr{W}=\Phi(\beta(\vec{f}) / \mathscr{W})$ iff $\{\langle i,[\Phi(\vec{f}(i))]\rangle \mid i \in I\} \in \mathcal{U}$ (by Lemma 7.1) iff $[\Phi(\beta(\vec{f}))] \in \mathcal{W}$, and by kos's Theorem this holds iff $\Pi_{\langle i, x\rangle \in I^{\prime}} A_{x}^{i} / \mathcal{W}=$ $\Phi(\beta(\vec{f}) /$ Q $)$.

We define the operator $\Gamma_{0}^{e}$ to be the operator $\Gamma_{T}^{e}$ (see $\left.\S 4\right)$ where $T$ is the theory of atomless Boolean algebras. Clearly $A[C]^{*} \in \Gamma_{0}^{e}(A)$, where $C$ is the Cantor discontinuum (a well-known Boolean space).

THEOREM 7.3. $\mathbf{S P}_{R}=\mathbf{S T}_{0}^{e} \mathbf{S}^{(<)} \mathbf{P}_{U}$.

Proof. Since $\mathbf{S P}_{R}=\mathbf{S P P}_{U}$ (by Theorem 2.1(d)) we only need to verify that $\mathbf{P} \leqslant \mathbf{S} \Gamma_{0}^{e} \mathbf{S}^{(\prec)} \mathbf{P}_{U}$. So suppose $\prod_{i \in I} A_{i}$ is given. Since $A_{i}$ is in $\operatorname{IS}\left(A_{i}[C]^{*}\right)$ it follows that $\prod_{i \in I} A_{i} \in \mathbf{I S}\left(\prod_{i \in I} A_{i}[C]^{*}\right)$. Let $X=\left(C^{* I}\right)^{*}$, and define $\alpha$ from $\prod_{i \in I} A_{i}[C]^{*}$ to $\prod_{\mathcal{Q} \in X}\left(\prod_{i \in I} A_{i}[C]^{*} / \mathcal{Q}\right)$ by $\alpha(f)(\mathcal{U})=f / \mathcal{Q}$. By Lemma 7.1, if $\Phi(\vec{u})$ is any $L$-formula and $\vec{f}$ is in $\prod_{i \in I} A_{i}[C]^{*}$ then $[\Phi(\alpha(\vec{f}))]=\{\mathcal{Q} \in f \mid\{\langle i,[\Phi(\vec{f}(i))]\rangle \mid i \in I\} \in \mathcal{U}\}$, and this suffices to establish the fact that $\alpha$ is an isomorphism and the image of $\alpha$ satisfies $\left(2^{e}\right)$. To verify (1) is, as usual, straightforward. For (3) suppose that $f, g \in \prod_{i \in I} A_{i}[C]^{*}$ and that $N$ is a clopen subset of $X$. Then we can find $\left\{\left\langle i, J_{i}\right\rangle \mid i \in I\right\} \in\left(C^{*}\right)^{I}$ 
such that for $\mathcal{U} \in X, \mathcal{U} \in N$ iff $\left\{\left\langle i, J_{i}\right\rangle \mid i \in I\right\} \in \mathcal{U}$. Define $h$ by $h(i)(x)$ $=f(i)(x)$ if $x \in J_{i}$, and $h(i)(x)=g(i)(x)$ if $x \in C-J_{i}$, for $i \in I$. Then $\{\langle i$, $\left.\left.J_{i}\right\rangle \mid i \in I\right\} \leqslant\{\langle i,[h(i)=f(i)]\rangle \mid i \in I\}$, so we have $\mathcal{Q} \in[\alpha(h)=\alpha(f)]$ if $\mathcal{Q} \in N$, and likewise $\mathcal{U} \in[\alpha(h)=\alpha(g)]$ if $\mathcal{U} \in X-N$, so $\alpha(h)=\alpha(f) \uparrow_{N}$ $\cup \alpha(g) \uparrow_{X-N} \cdot C^{* I}$ is certainly atomless, hence $\prod_{i \in I} A_{i}[C]^{*}$ belongs to $\mathbf{I} \Gamma_{0}^{e} \Gamma_{U}^{e}\left(\left\{A_{i} \mid i \in I\right\}\right)$, which in turn is contained in $\mathbf{I} \Gamma_{0}^{e} \mathbf{S}^{(<)} \mathbf{P}_{U}\left(\left\{A_{i} \mid i \in I\right\}\right)$ by Lemma 7.2(b). Thus we have $\prod_{i \in I} A_{i} \in \operatorname{IST}_{0}^{e} \mathbf{S}^{(<)} \mathbf{P}_{U}\left(\left\{A_{i} \mid i \in I\right\}\right)$, as was to be shown.

The following corollary is an addition to Theorem 4.5, and in particular it applies to $\Gamma_{0}^{e}$.

COROLlaRY 7.4. Let $T$ be a theory of Boolean algebras whose models $\Re(T)$ are closed under products, and whenever $B \vDash T$ and $F$ is a proper principal filter on $B$ then $B / F \vDash T$. Then

$$
\begin{aligned}
\operatorname{Th}\left(\Gamma_{T}^{e}(\Re)\right) & =\operatorname{Th}\left(\mathbf{P}\left(\left\{A[X]^{*} \mid A \in \Re, X^{*} \vDash T\right\}\right)\right) \\
& =\operatorname{Th}\left(\mathbf{P}_{\text {fin }}\left(\left\{A[X]^{*} \mid A \in \Re, X^{*} \vDash T\right\}\right)\right),
\end{aligned}
$$

where $\mathbf{P}_{\text {fin }}$ denotes closure under finite direct products, for any $\Re$.

Proof. Let $\mathfrak{G}=\left\{A[X]^{*} \mid A \in \mathfrak{\Re}, X^{*} \vDash T\right\}$. Since $\mathfrak{N}(T)$ is closed under products clearly $\mathbf{P} \Gamma_{T}^{e} \leqslant \Gamma_{T}^{e} \Gamma_{U}^{e}$ follows from looking at the construction used in the proof of Lemma 7.2(a). From Theorem 4.5(b) and Lemma 7.2(b) we have $\operatorname{Th}\left(\Gamma_{T}^{e}(\Re)\right)=\operatorname{Th}\left(\Gamma_{T}^{e} \Gamma_{U}^{e}(\Re)\right)$, hence $\operatorname{Th}\left(\Gamma_{T}^{e}(\Re)\right) \subseteq \operatorname{Th}\left(\mathbf{P r}_{T}^{e}(\Re)\right) \subseteq \operatorname{Th}(\mathbf{P}(\mathfrak{g}))$ $\subseteq \operatorname{Th}\left(\mathbf{P}_{\text {fin }}(\mathfrak{Q})\right)$. On the other hand, if $\Gamma_{T}^{e}(\Re) \not \Phi$ then, assuming we have a special determining sequence $\left\langle\Phi^{\prime} ; \Theta_{1}, \ldots, \Theta_{2^{\prime}}\right\rangle$ as in the proof of Theorem 4.5 , by Lemma 4.4 there is a $B=T$ and elements $b_{1}, \ldots, b_{m}$ in $B$ with $b_{1} \vee \cdots \vee b_{m}=1$ and $b_{i} \wedge b_{j}=0$ for $1 \leqslant i<j \leqslant m$, and $B$ F $\neg \Phi^{\prime}\left(b_{1}, \ldots, b_{m}, 0, \ldots, 0\right)$. Then, with $X_{i}=\left\{\mathscr{U} \in B^{*} \mid b_{i} \in \mathcal{U}\right\}$ and $A_{i} \models \Theta_{i}$, $A_{i} \in \Re$, note that $\Pi_{1 \leqslant i \leqslant m} A_{i}\left[X_{i}\right]^{*} \vDash \neg \Phi$. Because $X_{i}^{*}$ is isomorphic to a quotient of $B$ by a proper principal filter we have $X_{i}^{*} \vDash T$, hence $\mathbf{P}_{\text {fin }}(\mathfrak{G}) \not \neq \Phi$.

We define $\Gamma_{0}^{a}$ parallel to the definition of $\Gamma_{0}^{e}$, namely for any $\Re, \Gamma_{0}^{a}(\Re)=\{A$ $\in \Gamma^{a}(\Omega) \mid X(A)^{*}$ is atomless $\}$. From Theorem 7.3 and Theorem 2.1 we easily have the following.

COROllary 7.5. $\mathbf{S P}_{R}=\mathbf{S} \Gamma_{0}^{a} \mathbf{S}^{(<)} \mathbf{P}_{U}$.

8. Model companions for theories with few existential types. If $T$ is any set of sentences then $\mathfrak{M}(T)$ denotes the class of models of $T$, i.e. the class of all structures satisfying all the sentences in $T$. Two theories $T$ and $T^{*}$ are mutually model-consistent if $\mathbf{S M}(T)=\mathrm{SM}\left(T^{*}\right)$, i.e. every model of $T$ can be embedded in a model of $T^{*}$ and vice-versa. $T^{*}$ is model-complete if $A_{1}$, $A_{2} \in \mathfrak{M}\left(T^{*}\right)$ and $A_{1} \in \mathbf{S}\left(A_{2}\right)$ imply $A_{1} \prec A_{2}$. $T^{*}$ is the model companion of $T$ if (i) $T$ and $T^{*}$ are mutually model-consistent, and (ii) $T^{*}$ is model-complete. 
Let $E_{n}$ be the set of existential $L$-formulas $\exists \vec{u} \Phi(\vec{u}, \vec{v})$ with free variables contained in $\left\{v_{1}, \ldots, v_{n}\right\}$. A primitive formula is an existential formula of the form $\exists \wedge$ ( \pm atomic), where, of course, \pm atomic means a formula which is either atomic or negated atomic. Let $P_{n}=\left\{\Phi \in E_{n} \mid \Phi\right.$ is primitive $\}$. A model $A$ of $T$ is existentially closed in $T$ if for any given $\Phi(\vec{v})$ in $E_{n}$ and $\vec{a} \in A^{n}$, if there is a model $B$ of $T$ with $A$ a substructure of $B$ and $B=\Phi(\vec{a})$ then $A \vDash \Phi(\vec{a})$. (Clearly one can replace $E_{n}$ by $P_{n}$ in the definition of existentially closed.) We will need the following basic results on model companions (see [10] and [17]).

LEMMA 8.1. Let $T$ be a theory.

(a) $T$ is a model-complete theory iff for each $\Phi \in E_{n}, n<\omega$, there is a $\Psi \in E_{n}$ such that $T \vdash \Phi \leftrightarrow \neg \Psi$.

(b) If $T$ is an $\forall \exists$-axiomatizable theory then $T$ has a model companion iff the existentially closed models in $T$ form an elementary class (in which case the theory of the existentially closed models in $T$ is the model companion).

One of the key tools for analyzing existentially closed structures in this and the next section is the following result.

LEMMA 8.2 (MACINTYRE). If $A \in \Gamma_{0}^{e}(\Re)$ and $\Phi(\vec{v}) \in P_{n}$, say $\Phi(\vec{v})$ is $\exists \vec{u} \mid \Phi_{0}(\vec{u}$, $\left.\vec{v}) \& \&_{1 \leqslant i \leqslant k} \neg \alpha_{i}(\vec{u}, \vec{v})\right]$ where $\Phi_{0}(\vec{u}, \vec{v})$ is a conjunct of atomic formulas, and each $\alpha_{i}$ is atomic, then for $\vec{f} \in A: A=\Phi(\vec{f})$ iff

(i) $\left[\exists \vec{u} \Phi_{0}(\vec{u}, \vec{f})\right]=X(A)$ and

(ii) $\left[\exists \vec{u}\left(\Phi_{0}(\vec{u}, \vec{f}) \& \neg \alpha_{i}(\vec{u}, \vec{f})\right)\right] \neq \varnothing, 1 \leqslant i \leqslant k$.

Proof. $(\Rightarrow)$ This direction is straightforward.

$(\Leftarrow)$ Using the Maximal Property choose sequences of elements $\vec{g}^{(0)}, \ldots, \vec{g}^{(k)}$ from $A$ such that

(i') $\left[\Phi_{0}\left(\vec{g}^{(0)}, \vec{f}\right)\right]=X(A)$ and

(ii') $\left[\Phi_{0}\left(\vec{g}^{(i)}, \vec{f}\right) \& \neg \alpha_{i}\left(\vec{g}^{(i)}, \vec{f}\right)\right] \neq \varnothing, 1 \leqslant i \leqslant k$.

Since $X(A)$ has no isolated points choose pairwise disjoint, nonempty clopen subsets $N_{1}, \ldots, N_{k}$ with $N_{i} \subseteq\left[\Phi_{0}\left(\vec{g}^{(i)}, f\right) \& \neg \alpha_{i}\left(\vec{g}^{(i)}, \vec{f}\right)\right]$. Then, with $\vec{g}=$ $\vec{g}^{(0)} \uparrow_{\left(X-U_{1<i<k} N_{i}\right)} \cup \cup_{1 \leqslant i \leqslant k} \vec{g}^{(i)} \uparrow_{N_{i}}$, we have

$\left(\mathrm{i}^{\prime \prime}\right)\left[\Phi_{0}(\bar{g}, f)\right]=X(A)$ and

(ii") $\left[\Phi_{0}(\vec{g}, \vec{f}) \&-\alpha_{i}(\vec{g}, \vec{f})\right] \neq \varnothing, 1 \leqslant i \leqslant k$, and hence $A=\Phi_{0}(\vec{g}, \vec{f}) \&_{1 \leqslant i \leqslant k} \neg \alpha_{i}(\vec{g}, \vec{f})$, so $A \vDash \Phi(\vec{f})$.

Given a theory $T$ let $\varepsilon_{n}(T)=k$ if, modulo $T$, there are exactly $k$ inequivalent members of $P_{n}$.

Lemma 8.3. Given $T$ let $T^{\prime}=\operatorname{Th}\left(\Gamma_{0}^{e} \mathfrak{M}(T)\right)$. Then

(a) $\varepsilon_{n}(T)<\omega \Rightarrow \varepsilon_{n}\left(T^{\prime}\right)<\omega$, for $n<\omega$,

(b) $\varepsilon_{0}(T)=2 \Rightarrow \varepsilon_{0}\left(T^{\prime}\right)=2$.

Proof. This is an easy consequence of Lemma 8.2. 
THEOREM 8.4. Given $T$ let $T^{\prime \prime}=\operatorname{Th}\left(\mathbf{S P}_{R} \mathfrak{M}(T)\right)$.

(a) If $\varepsilon_{n}(T)<\omega$ for $n<\omega$ then $T^{\prime \prime}$ has a model companion, and

(b) if in addition $\varepsilon_{0}(T)=2$ then the model companion is $\boldsymbol{\aleph}_{0}$-categorical, provided the language is countable and $T$ has a model with at least two elements in its universe.

Proof. (a) Let $T^{\prime}=\operatorname{Th}\left(\Gamma_{0}^{e} \mathfrak{M}(T)\right)$. From Theorem 7.3, $\mathfrak{M}\left(T^{\prime \prime}\right)=\operatorname{SM}\left(T^{\prime}\right)$. Also, by Lemma 8.3 we have $\varepsilon_{n}\left(T^{\prime}\right)<\omega$ for $n<\omega$, hence for $\Phi(\vec{v}) \in P_{n}$ it is possible to choose $\Phi^{*}(\vec{v}) \in E_{n}$ such that

$$
T^{\prime} \vdash \Phi^{*}(\vec{v}) \leftrightarrow \bigvee\left\{\Psi(\vec{v}) \in P_{n} \mid T^{\prime} \vdash \Psi(\vec{v}) \rightarrow \neg \Phi(\vec{v})\right\}
$$

Now we claim: $A$ is existentially closed in $T^{\prime \prime}$ iff

$$
A \vDash T^{\prime \prime} \cup\left\{\forall \vec{v}\left(\Phi(\vec{v}) \vee \Phi^{*}(\vec{v})\right) \mid \Phi(\vec{v}) \in P_{n}\right\} .
$$

So suppose $A$ is existentially closed in $T^{\prime \prime}$, that $\Phi(\vec{v}) \in P_{n}$ and $\vec{a} \in A$. If $A \vDash \neg \Phi(\vec{a})$, let $D(A)$ be the open diagram of $A$ and note that $T^{\prime} \cup D(A) \cup$ $\{\Phi(\vec{a})\}$ is inconsistent, so $T^{\prime} \vdash \Psi(\vec{v}) \rightarrow \neg \Phi(\vec{v})$ for some $\Psi \in P_{n}$ with $A$ 乑 $\Psi(\vec{a})$. Then $T^{\prime} \vdash \Psi(\vec{v}) \rightarrow \Phi^{*}(\vec{v})$, hence $A \models \Phi^{*}(\vec{a})$. Conversely, if $A \models T^{\prime \prime} \cup$ $\left\{\forall \vec{v}\left(\Phi(\vec{v}) \vee \Phi^{*}(\vec{v})\right) \mid \Phi(\vec{v}) \in P_{n}\right\}$ suppose $\Phi(\vec{v}) \in P_{n}$, assume $B=T^{\prime \prime}$ with $A$ a substructure of $B$ and $B \vDash \Phi(\vec{a})$. We can, without loss of generality, assume $B \vDash T^{\prime}$, hence $B \vDash \neg \Phi^{*}(\vec{a})$, so $A \vDash \neg \Phi^{*}(\vec{a})$, and then $A \vDash \Phi(\vec{a})$. Now apply Lemma 8.1(b).

(b) Let $T^{*}$ be the model companion of $T^{\prime}$ (or equivalently of $T^{\prime \prime}$ ). Since $\varepsilon_{n}\left(T^{\prime}\right)<\omega$ for $n<\omega$ it follows that $\varepsilon_{n}\left(T^{*}\right)<\omega$ for $n<\omega$ as $\varepsilon_{n}\left(T^{*}\right) \leqslant \varepsilon_{n}\left(T^{\prime}\right)$. Modulo $T^{*}$ every formula is equivalent to an existential formula (Lemma 8.1(a)), hence for each $n<\omega$ there are only finitely many $n$-types consistent with $T^{*}$, and since $\varepsilon_{0}\left(T^{*}\right)=2$ it follows that $\operatorname{Th}\left(T^{*}\right)$ is complete. The condition that $T$ have a model with at least two elements in it ensures that $T^{\prime}$ has infinite models. So by Ryll-Nardzewski's result it follows that $T^{*}$ is $\aleph_{0}$-categorical.

COROLlaRY 8.5. If $\Re$ is a finite collection of finite structures, or $\mathrm{Th}(\Re)$ is a countable $\aleph_{0}$-categorical theory without finite models, then $\operatorname{Th}\left(\mathbf{S P}_{R}(\Re)\right)$ has a model companion (which is $\boldsymbol{\aleph}_{0}$-categorical in case $\mathrm{Th}(\Re)$ is complete and countable).

An algebra $A$ is a structure without fundamental relations. For any class $\Re$ of algebras let $\mathbf{V}(\Re)$ be the variety generated by $\Re$. The following observation is useful in applying Corollary 8.5 .

Proposition 8.6. Let $A$ be an algebra such that every finitely generated nontrivial subdirectly irreducible algebra in $\mathrm{V}(A)$ can be embedded into $A$. Then $\mathbf{I S P}_{R}(A)=\mathbf{V}(A)$. 
Proof. From results to Mal'cev [29] we know that every algebra can be embedded into an ultraproduct of its finitely generated subalgebras, and since finitely generated subalgebras are subdirect products of finitely generated subdirectly irreducible algebras the result follows.

Combining Corollary 8.5 and Proposition 8.6 we can conclude that the following list of varieties have $\boldsymbol{\kappa}_{0}$-categorical model companions: any variety generated by a finite lattice, Heyting algebra, or abelian group; the variety of semilattices with pseudo-complementation; any variety of monadic algebras (Comer [14]), distributive lattices with pseudo-complementation, and relative Stone algebras. The reader will easily think of other examples. We do not know if there is a model companion for the theory of any variety generated by a finite algebra.

A natural question in this search for model companions is: when is $\mathrm{Th}\left(\boldsymbol{\Gamma}_{0}^{e}(\Omega)\right)$ model-complete? Some sufficient conditions are given in $\S 9$ and $\S 10$, but for now we can say that if $\Re=\{A\}$ and $A$ is a finite structure with at least two elements, or $\operatorname{Th}(A)$ is countable, $\aleph_{0}$-categorical, and $A$ is infinite, then from Theorem 4.5(f) it follows that $\operatorname{Th}\left(\Gamma_{0}^{e}(\Re)\right)$ is $\kappa_{0}$-categorical, hence if it is also $\forall \exists$-axiomatizable then by Lindström [25] it must be model-complete.

9. Discriminator varieties. In this section we take a brief look at discriminator varieties ${ }^{10}$ and develop most of the results needed for discriminator formulas in the next section. Recalling the definition of $E_{n}$ and $P_{n}$ in $\$ 8$ let $E_{n}^{+}$be the positive formulas in $E_{n}$, and then define $P_{n}^{+}$to be $P_{n} \cap E_{n}^{+}$(hence the members of $P_{n}^{+}$are primitive positive). For $T$ a theory of $L$-structures and $A$ a model of $T, A$ is said to be algebraically closed in $T$ if $A \models \Phi(\vec{a})$ for every $\Phi(\vec{v}) \in E_{n}^{+}, n<\omega$, and $\vec{a} \in A$ such that some model $B$ of $T$ extending $A$ satisfies $\Phi(\vec{a})$. (Clearly it is enough to look only at the $\Phi(\vec{v})$ in $P_{n}^{+}$.)

For the remainder of this section we will assume that our language $L$ is a language of algebras (i.e. a language without relation symbols), and that $t$ is a new symbol for a ternary operation, the expanded language being $L(t)$. If $A$ is an algebra let $A^{t}$ denote the expansion of $A$ defined by:

$$
A^{t} \vDash t(u, v, w)=z \leftrightarrow(u=v \& w=z) \vee(u \neq v \& u=z),
$$

and then for $\Re$ a class of $L$-algebras let $\Re^{t}=\left\{A^{t} \mid A \in \Re\right\}$. Define $n\left(u_{1}, u_{2}, v_{1}\right.$, $\left.v_{2}\right)$ to be the term $t\left(t\left(u_{1}, u_{2}, v_{1}\right), t\left(u_{1}, u_{2}, v_{2}\right), v_{2}\right)$. One easily checks that

$$
\begin{aligned}
& A^{t} \vDash n\left(u_{1}, u_{2}, v_{1}, v_{2}\right)=w \leftrightarrow\left(u_{1}=u_{2} \& v_{1}=w\right) \vee\left(u_{1} \neq u_{2} \& v_{2}=w\right), \\
& A^{t} \vDash t(u, v, w)=n(u, v, w, u) .
\end{aligned}
$$
[42].

${ }^{10}$ For a comprehensive treatment of discriminator varieties the reader is referred to Werner 
From these definitions it is straightforward to verify:

LEMMA 9.1. For any class of algebras $\mathfrak{\Re}$,

$$
\begin{aligned}
& \AA^{t} \vDash\left(u_{1}=u_{2} \bigvee v_{1}=v_{2}\right) \leftrightarrow n\left(u_{1}, u_{2}, v_{1}, v_{2}\right)=v_{1}, \\
& \Omega^{t} \vDash\left(u_{1}=u_{2} \& v_{1}=v_{2}\right) \leftrightarrow t\left(u_{1}, u_{2}, v_{1}\right)=t\left(u_{2}, u_{1}, v_{2}\right), \\
& \Omega^{t} \vDash\left(u_{1}=u_{2} \bigvee v_{1} \neq v_{2}\right) \leftrightarrow n\left(v_{1}, v_{2}, u_{1}, u_{2}\right)=u_{2} .
\end{aligned}
$$

We will use \#A in the following for the cardinality of $A$.

LemMA 9.2. For any L-formula $\Phi(\vec{v})$, say $\Phi(\vec{v})$ is $\overrightarrow{Q u} \Psi(\vec{u}, \vec{v})$ where $\overrightarrow{Q u}$ is a string of quqntified variables and $\Psi(\vec{u}, \vec{v})$ is open, there is an atomic $L(t)$-formula $\hat{\Psi}\left(w_{1}, w_{2}, \vec{u}, \vec{v}\right)$ such that, with $\Phi^{*}(\vec{v})$ defined as $\forall w_{1} \forall w_{2} \overrightarrow{Q u} \hat{\Psi}\left(w_{1}, w_{2}, \vec{u}\right.$, $\vec{v})$, one has for any algebra $A$ and any $\vec{a}$ in $A$ :

$$
A^{t} \vDash \Phi^{*}(\vec{a}) \quad \text { iff } \# A=1 \text { or } A=\Phi(\vec{a}) .
$$

Proof. First use Lemma 9.1 to show that there is an atomic formula $p(\vec{u}, \vec{v})=q(\vec{u}, \vec{v})$ in the language $L(t)$ such that $A^{t} \vDash \Psi(\vec{u}, \vec{v}) \leftrightarrow p(\vec{u}, \vec{v})=$ $q(\vec{u}, \vec{v})$ or $A^{t} \vDash \Psi(\vec{u}, \vec{v}) \leftrightarrow p(\vec{u}, \vec{v}) \neq q(\vec{u}, \vec{v})$. In the first case let $\hat{\Psi}\left(w_{1}, w_{2}, \vec{u}\right.$, $\vec{v})$ be $n\left(w_{1}, w_{2}, p(\vec{u}, \vec{v}), q(\vec{u}, \vec{v})\right)=p(\vec{u}, \vec{v})$, and in the second case let it be $n\left(p(\vec{u}, \vec{v}), q(\vec{u}, \vec{v}), w_{1}, w_{2}\right)=w_{2}$. Then, for $b_{1}, b_{2} \in A, A^{t}=\overrightarrow{Q u} \hat{\Psi}\left(b_{1}, b_{2}, \vec{u}, \vec{a}\right)$ iff $b_{1}=b_{2}$ or $A^{t} \vDash \overrightarrow{Q u} \Psi(\vec{u}, \vec{a})$. Thus $A=\forall w_{1} \forall w_{2} \overrightarrow{Q u} \hat{\Psi}\left(w_{1}, w_{2}, \vec{u}, \vec{a}\right)$ iff $\# A=1$ or $A \vDash \overrightarrow{Q u} \Psi(\vec{u}, \vec{a})$.

The next lemma shows that formulas in ${P_{n}^{+}}^{+}$behave like atomic formulas with respect to $\Gamma^{a}(\Re)$.

Lemma 9.3. Suppose $\Phi(\vec{v}) \in P_{n}^{+}, A \in \Gamma^{a}(\Re)$ and $\vec{a} \in A$. Then

$$
A \models \Phi(\vec{a}) \quad \text { iff } X(A)=[\Phi(\vec{a})] .{ }^{11}
$$

Proof. $(\Rightarrow)$ Note that $\Phi(\vec{a})$ is positive.

$(\Leftarrow)$ Suppose $\Phi(\vec{v})$ is $\exists \vec{u} \Phi^{\prime}(\vec{u}, \vec{v})$ with $\Phi^{\prime}(\vec{u}, \vec{v})$ a conjunct of atomic formulas. By the Patchwork Property there is a $\vec{g}$ such that $X(A)=\left[\Phi^{\prime}(\vec{g}, \vec{f})\right]$. But then $A=\Phi^{\prime}(\vec{g}, \vec{f})$, so $A \vDash \Phi(\vec{f})$.

If $\boldsymbol{\Sigma}$ is a set of $L$-statements let $\boldsymbol{\Sigma}^{*}=\left\{\Phi^{*} \mid \Phi \in \boldsymbol{\Sigma}\right\}$, where $\Phi^{*}$ is as in Lemma 9.2. For $\Omega$ a class of algebras let $\Omega_{+}$be the class $\Omega$ plus the one-element algebras.

LEMMA 9.4. Let $\Re$ be an elementary class such that $\mathrm{Th}(\Re)$ has a set $\mathbf{\Sigma}$ of $\forall \exists$ axioms. Then

$$
\mathbf{I} \Gamma^{a}\left({\Omega_{+}^{t}}_{+}\right)=\left\{A \in \mathbf{I S} \Gamma^{a}\left(\Omega_{+}^{t}\right) \mid A \vDash \mathbf{\Sigma}^{*}\right\},
$$

\footnotetext{
"Another way of stating this result is that for $A \in \Gamma^{a}(\Re)$ the natural embedding $A \rightarrow$ $\Pi_{x \in X(A)} A_{x}$ is pure. This was used by Bulman-Fleming and Werner to describe the equationally compact algebras in residually finite discriminator varieties in [5]. (Some properties of these algebras were known to Taylor [36].)
} 
and if $\boldsymbol{\Sigma}$ is a set of universal sentences then

$$
\mathbf{I} \Gamma^{a}\left(\Re_{+}^{t}\right)=\operatorname{IST}^{a}\left(\Omega_{+}^{t}\right) .
$$

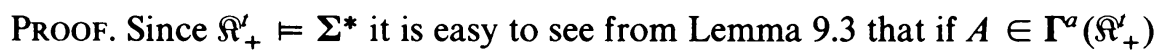
then $A \vDash \Sigma^{*}$ (as the sentences in $\Sigma^{*}$ are of the form $\forall \exists$ (atomic)). So suppose $A \in \mathbf{S} \Gamma^{a}\left(\Omega_{+}^{t}\right)$, say $A$ is a subalgebra of $B$ where $B \in \Gamma^{a}\left(\Omega_{+}^{t}\right)$. Let $Z(A)=\{[f$ $=g] \mid f, g \in A\} \cup\{[f \neq g] \mid f, g \in A\}$. By Lemma 9.1, $Z(A)$ is a subalgebra of $X(B)^{*}$. For $थ \in Z(A)^{*}$ let $\sim_{\mathcal{Q}}$ be the relation defined on $A$ by $f \sim_{\mathcal{Q}} g$ iff [ $f=g] \in \mathcal{U}$, hence iff $\cap \mathcal{Q} \subseteq[f=g]$. This is clearly a congruence on $A$, and the mapping $\alpha: A / \sim_{\mathscr{Q}} \rightarrow B_{x}$, for any given $x \in \cap \mathcal{Q}$, defined by $\alpha\left(f / \sim_{\mathcal{Q}}\right)=f(x)$, is an embedding since for $f, g$ in $A$, either $\cap \mathcal{Q} \subseteq[f=g]$ or else $\cap \mathcal{Q} \subseteq[f \neq g]$. But then $A / \sim_{\mathcal{Q}} \vDash t(u, v, w)=z \leftrightarrow(u=v \& w=z)$ $\vee(u \neq v \& u=z)$. Now if $A=\Sigma^{*}$ then $A / \sim_{\mathcal{Q}}=\Sigma^{*}$ as all the sentences in $\Sigma^{*}$ are positive, hence by Lemma $9.2 \# A / \sim_{\mathcal{Q}}=1$ or $A / \sim_{\mathcal{Q}}=\Sigma$, i.e. $A / \sim_{\mathcal{Q}} \in$ $\Omega_{+}^{t}$. Now look at the mapping $\beta: A \rightarrow \Pi_{\mathscr{Q} \in Z(A)^{*}} A / \sim_{\mathcal{Q}}$ defined by $\beta(f)(\mathcal{Q})$ $=f / \sim_{2}$. This is readily seen to be an embedding, and $\beta(A)$ satisfies (1). For $f, g \in A,[\beta(f)=\beta(g)]=\{\mathscr{Q} \mid[f=g] \in \mathscr{U}\} \in Z(A)^{* *}$, so $\beta(A)$ satisfies $\left(2^{a}\right)$. Finally, for $f, g \in A$ and $N \in Z(A)^{* *}$, (by symmetry) we can assume that $N$ is $\left\{\mathscr{Q} \in Z(A)^{*} \mid[h=k] \in \mathcal{Q}\right\}$ for some $h, k \in A$ to show that $\beta(A)$ satisfies (3), namely $\beta(f) \uparrow_{N} \cup \beta(g) \uparrow_{z(A)^{*}-N}=\beta(n(h, k, f, g))$. If we assume that $\boldsymbol{\Sigma}$ is a set of universal sentences, then in the above proof we can conclude $A / \sim_{\mathcal{Q}} \in \Omega_{+}^{t}$ without the assumption $A \vDash \Sigma^{*}$. This yields the second equality.

Corollary 9.5. Let $\Re$ be an elementary class with a set $\Sigma_{1}$ of $\forall \exists$ axioms, and let $\boldsymbol{\Sigma}_{0}$ be a set of axioms for $\operatorname{Th}\left(\mathbf{S P}\left(\Omega_{+}^{t}\right)\right)$. Then $\mathbf{I} \Gamma^{a}\left(\Omega_{+}^{t}\right)$ is an elementary class whose theory is axiomatized by $\boldsymbol{\Sigma}_{0} \cup \boldsymbol{\Sigma}_{1}^{*}$.

Proof. From Theorem 2.1(d) we know $\operatorname{ISS}^{a}\left(\Re_{+}^{t}\right)=\operatorname{ISP}\left(\Re_{+}^{t}\right)$. Just combine this fact with Lemma 9.4.

Next we look at some of the important facts about the congruences of members of $\Gamma^{a}\left(\Omega^{\prime}\right)$. If $a, b \in A$ let $\theta(a, b)$ be the principal congruence generated by $\langle a, b\rangle$, i.e. the smallest congruence of $A$ containing $\langle a, b\rangle$. If $A \in \Gamma^{a}(\Re)$ and $Y \neq \varnothing$ is a closed subset of $X(A)$ let $A \uparrow_{Y}$ be the subalgebra of $\prod_{x \in Y} A_{x}$ with universe $\left\{f \uparrow_{Y} \mid f \in A\right\}$. Since closed subsets of Boolean spaces are Boolean spaces it is easy to check that $A \uparrow_{Y} \in \Gamma^{a}(\Re)$, and $X\left(A \uparrow_{Y}\right)$ $=Y$.

Lemma 9.6. Let $A \in \Gamma^{a}\left(\Omega^{t}\right)$.

(a) For $h, k \in A$,

$$
\begin{aligned}
\theta(h, k) & =\left\{\langle f, g\rangle \in A^{2} \mid[h=k] \subseteq[f=g]\right\} \\
& =\left\{\langle f, g\rangle \in A^{2} \mid n(h, k, f, g)=g\right\} .
\end{aligned}
$$


(b) Every finitely generated congruence of $A$ is principal.

(c) If $Y$ is a closed subset of $A$ let $\theta_{Y}=\left\{\langle f, g\rangle \in A^{2} \mid Y \subseteq[f=g]\right\}$. Then $\theta_{Y}$ is a congruence on $A$; and $A / \theta_{Y} \cong A \uparrow_{Y}$ under the map $f / \theta_{Y} \rightarrow \uparrow_{\uparrow_{Y}}$, if $Y \neq \varnothing$.

(d) If $\theta$ is a congruence on $A$ let $Y=\bigcap_{\langle f, g\rangle \in \theta}[f=g]$. Then $\theta=\theta_{Y}$.

Proof. (a) Clearly $\langle f, g\rangle \in \theta(h, k)$ implies $[h=k] \subseteq[f=g]$, hence $n(h, k, f, g)=g$ by Lemma 9.1. Conversely, $n(h, k, f, g)=g$ implies [ $h=k]$ $\subseteq[f=g]$, and from this $\langle n(h, h, f, g), n(h, k, f, g)\rangle \in \theta(h, k)$ implies $\langle f, g\rangle \in \theta(h, k)$.

(b) Note that for $f, g, h, k \in A$,

$$
\theta(f, g) \vee \theta(h, k)=\theta(t(f, g, h), t(g, f, k)) .
$$

(c) This is straightforward.

(d) Since $\theta=\bigcup_{\langle h, k\rangle \in \theta} \theta(h, k)$ it follows from (a) that $\theta \subseteq \theta_{Y}$. On the other hand if $\langle f, g\rangle \in \theta_{Y}$ then, since $[f \neq g]$ is compact and the set $\{\theta(h, k) \mid\langle h, k\rangle \in \theta\}$ is directed (by (b)), $[h=k] \subseteq[f=g]$ for some $\langle h, k\rangle \in$ $\theta$, hence by (a) $\langle f, g\rangle \in \theta$.

An important consequence of Lemma 9.6(d) is that every $A \in \mathbb{\Omega}^{\ell}$ with $\# A>1$ is simple.

Next we give the representation theorem referred to in $\S 6$. Recall that $V(\Re)$ is the variety generated by $\Omega$. Up to polynomial equivalence the discriminator varieties are the $\mathbf{V}\left(\Omega^{t}\right)$.

THEOREM 9.7. Let $\Re$ be any class of L-algebras.

(a) (Bulman-Fleming, Keimel, Werner), $\mathbf{V}\left(\Re^{t}\right)=\mathbf{I}{ }^{a} \mathbf{S P}{ }_{U}\left({\Re_{+}^{t}}^{t}\right)$.

(b) If no member of $\mathbf{P}_{U}(\Re)$ properly contains a one-element subalgebra then $\mathbf{V}\left(\Re^{t}\right)=\left(\mathbf{I}{ }^{a} \mathbf{S} \mathbf{P}_{U}\left(\Omega^{t}\right)\right)_{+}$.

Proof. (a) Clearly $I \Gamma^{a} \mathbf{S P}{ }_{U}\left(\Re_{+}^{t}\right) \subseteq \mathbf{V}\left(\Omega^{t}\right)$, and from Lemma 9.4, Ir $^{a} \mathbf{S P}_{U}\left(\Omega_{+}^{t}\right)=\mathbf{I S \Gamma} \Gamma^{a} \mathbf{S P}_{U}\left(\Omega_{+}^{t}\right)$, which in turn equals $\mathbf{I S P}_{\boldsymbol{R}}\left(\Omega_{+}^{t}\right)$ by Theorem 2.1. So we only need to show that $\operatorname{Ir}^{a} \mathbf{S P}_{U}\left({\Omega^{t}}_{+}\right)$is closed under quotients. If $\theta$ is a congruence on $A$ and $A \in \Gamma^{a} \mathbf{S P}_{U}\left(\Omega_{+}^{t}\right)$, then $\theta=\theta_{Y}$ for some closed subset $Y$ of $X(A)$ by Lemma 9.6(d), and then $A / \theta \in \mathbf{I \Gamma}^{a} \mathbf{S P}_{U}\left(\Omega_{+}^{t}\right)$ by Lemma 9.6(c).

(b) For such a $\Re$ an easy compactness argument shows that there must be finitely many fundamental operations, say $\mathbf{f}_{1}, \ldots, \mathbf{f}_{n}$, such that for any $A \in \mathbf{S P}_{U}\left(\Omega_{+}^{t}\right)$ and $a \in A$, if $\# A>1$ then $\mathbf{f}_{i}(a, \ldots, a) \neq a$ for some $i$, $1 \leqslant i \leqslant n$. Now suppose $B \in \Gamma^{a} \mathbf{S P}_{U}\left(\Re_{+}^{t}\right)$ and $\# B>1$. Let $f$ be any fixed member of $B$. Then $[\forall u \forall v(u=v)]=\left[\&_{1 \leqslant i \leqslant n} f_{i}(f, \ldots, f)=f\right]$, hence [ $\forall u \forall v(u=v)]$ is a clopen subset $N$ of $X(B)$. But then it is easy to see that $B \cong B \uparrow_{X(B)-N} \in \Gamma^{a} \mathbf{S P}_{U}\left(\Omega^{t}\right)$. 
LEMMA 9.8. Let $\Omega$ be a class of L-algebras.

(a) If $\mathrm{Th}(\Omega)$ is model-complete then $\Gamma^{a}(\Re)=\Gamma^{e}(\Re)$.

(b) If $\mathrm{Th}(\Re)$ is model-complete then $\mathrm{Th}\left(\Omega^{t}\right)$ is model-complete.

(c) If $\mathrm{Th}(\Omega)$ is decidable then $\mathrm{Th}\left(\Omega^{t}\right)$ is decidable.

Proof. (a) If $\Phi \in E_{0}$ and $A \in \Gamma^{a}(\Re)$ then it is easy to see that [ $\left.\Phi\right]$ is an open subset of $X(A)$ (actually for any $\Re$ ). Thus, applying Lemma 8.1(a) we see that for any $L$-sentence $\Phi,[\Phi]$ is an open subset of $X(A)$, hence $A \in \Gamma^{e}(\Re)$.

(b) \& (c) Merely note that $t$ is definable in $\Re^{t}$ by an open $L$-formula.

Now we will look at some examples of residually large varieties (indeed these varieties have arbitrarily large simple algebras in them) which have decidable theories, as promised in Remark 4 of $\$ 6$. Makowsky [28] notes that from a finitely generated infinite group $G$ one can construct a universal, complete, and model-complete theory $T_{G}$ of algebras with finitely many unary operations, and no model of $T_{G}$ has a one-element subalgebra. One can easily check that $T_{G}$ has a decidable theory iff $G$ has a solvable word problem. For $G$ a finitely generated infinite group with a solvable word problem let $\mathfrak{M}\left(T_{G}\right)$ be $\Re$. From Theorem 9.7(b), $\mathbf{V}\left(\Re^{t}\right)=\left(I \Gamma^{a}\left(\Omega^{t}\right)\right)_{+}$, and then by Lemma 9.8(a), (b) this is $\left(I \Gamma^{e}\left(\Omega^{t}\right)\right)_{+}$. Lemma 9.8(c) and Theorem 4.5(a) show that $\operatorname{Th}\left(\Gamma^{e}\left(\Omega^{t}\right)\right.$ ) is decidable, hence $\operatorname{Th}\left(\mathbf{V}\left(\Omega^{t}\right)\right.$ ) is also decidable. (As noted above, every algebra in $\Omega^{t}$ is simple.)

Lemma 9.9. If $A, B \in \mathbf{I} \Gamma^{a}\left(\Omega^{t}\right)$ and $A$ is a subalgebra of $B$ then, for $\theta$ a congruence of $A$, there is a congruence $\theta^{\prime}$ of $B$ such that $\theta=\theta^{\prime} \cap A^{2}$.

Proof. For $a, b \in A$ let $\theta_{A}(a, b)$, respectively $\theta_{B}(a, b)$, be the principal congruence of $A$, respectively $B$, generated by $\langle a, b\rangle$. Now $\theta=$ $\cup_{\langle a, b\rangle \in \theta} \theta_{A}(a, b)$, and this union is directed by Lemma 9.6(b). Let $\theta^{\prime}=$ $\cup_{\langle a, b\rangle \in \theta} \theta_{B}(a, b)$. Then this union is also directed as $\theta_{A}(a, b) \subseteq \theta_{A}\left(a^{\prime}, b^{\prime}\right)$ iff $n\left(a^{\prime}, b^{\prime}, a, b\right)=b$ iff $\theta_{B}(a, b) \subseteq \theta_{B}\left(a^{\prime}, b^{\prime}\right)$ by Lemma 9.6(a), and hence $\theta^{\prime}$ is a congruence of $B$. Now

$$
\begin{aligned}
\theta_{A}(a, b) & =\left\{\langle c, d\rangle \in A^{2} \mid n(a, b, c, d)=d\right\} \\
& =\left\{\langle c, d\rangle \in B^{2} \mid n(a, b, c, d)=d\right\} \cap A^{2}=\theta_{B}(a, b) \cap A^{2} .
\end{aligned}
$$

Hence $\theta=\theta^{\prime} \cap A^{2}$.

Lemma 9.10. Let $\Re$ be a class of algebras. If $A, B \in \Gamma^{a}\left(\Omega^{t}\right)$ and $\alpha: A \rightarrow B$ is an embedding then for any $x \in X(A)$ such that $\# A_{x}>1$ there is a $y \in X(B)$ for which $\beta: A_{x} \rightarrow B_{y}$ defined by $\beta(f x)=(\alpha f)(y), f \in A$, is an embedding.

Proof. Let $\theta_{\{x\}}$ be as defined in Lemma 9.6(c), and let $\theta=\{\langle\alpha f, \alpha g\rangle \mid$ $\left.\langle f, g\rangle \in \theta_{\{x\}}\right\}$. Then $\theta$ is a congruence on $\alpha(A)$, and by Lemma 9.9 there is a congruence $\theta^{\prime}$ on $B$ such that $\theta=\theta^{\prime} \cap(\alpha A)^{2}$. By Lemma 9.6(d) there is a 
closed subset $Y$ of $X(B)$ such that $\theta^{\prime}=\left\{\langle f, g\rangle \in B^{2} \mid Y \subseteq[f=g]\right\}$, so $\theta=\left\{\langle f, g\rangle \in(\alpha A)^{2} \mid Y \subseteq[f=g]\right\}$. Obviously $\alpha(A) / \theta \cong A / \theta_{\{x\}}$, and from Lemma 9.6(a) $A / \theta_{\{x\}} \cong A_{x}$, hence \# $\alpha(A) / \theta>1$. Consequently, for some $y \in Y$ there is an $\langle f, g\rangle \in(\alpha A)^{2}$ with $f(y) \neq g(y)$. For this $y$ we have $\# B_{y}>1$; and $y \in Y$ implies $f(y)=g(y)$ if $\langle f, g\rangle \in \theta$. The map $\beta: A_{x} \rightarrow B_{y}$ above is indeed well defined, for if $f, g \in A$ and $f(x)=g(x)$ then $\langle f, g\rangle \in \theta_{\{x\}}$, so $\langle\alpha f, \alpha g\rangle \in \theta$, hence $(\alpha f)(y)=(\alpha g)(y)$. But then $\beta$ is a homomorphism, and $\# \beta\left(A_{x}\right)>1$. From Lemma 9.6(d) $A_{x}$ is a simple algebra, hence $\beta$ is an embedding.

LEMMA 9.11. Let $\chi$ be the sentence:

$$
\forall u_{1} \forall u_{2} \exists v_{1} \exists v_{2}\left[n\left(u_{1}, u_{2}, v_{1}, v_{2}\right) \neq v_{2}\right] .
$$

Then for $A \in \Gamma^{a}\left(\Omega^{t}\right), A \vDash \chi$ iff $[\forall u \forall v(u=v)]$ is not a clopen subset of $X(A)$.

Proof. Using the Patchwork Property it is easy to verify that for $A \in$ $\Gamma^{a}\left(\Omega^{a}\right),[\forall u \forall v(u=v)]$ is clopen iff there are $f, g \in A$ such that $[f \neq g]=$ [ $\exists u \exists v(u \neq v)$ ]. However note that $A \vDash \chi$ iff for every $f, g \in A$ there is an $h$, $k \in A$ such that $[h \neq k] \underline{\&}[f \neq g]$.

LEMMA 9.12. Let $\Pi$ be the sentence:

$$
\forall u \forall v \exists w[u \neq v \rightarrow(u \neq w) \& n(u, v, u, w)=w \& n(u, w, u, v) \neq v] .
$$

Then for $A \in \Gamma^{a}\left(\Omega^{t}\right), A \vDash \Pi$ iff there is a $B \in \Gamma^{a}\left(\AA^{t}\right)$ such that $A \cong B$ and $X(B)^{*}$ is atomless.

Proof. Let $A \in \Gamma^{a}\left(\Omega^{t}\right)$. If $\# A=1$ then select any $x \in X(A)$ and let $B=A_{x}[C]^{*}$. If $\# A>1$ let $B^{\prime}$ be the $\beta(A)$ defined in the proof of Lemma 9.4. Then $A \cong B^{\prime}$ and every clopen subset of $X\left(B^{\prime}\right)$ is of the form [ $\left.f=g\right]$ or $[f \neq g]$. If $[\forall u \forall v(u=v)]_{B^{\prime}}$ is not clopen let $B=B^{\prime}$; and if $N=[\forall u \forall v(u=$ $v)]_{B^{\prime}}$ is clopen let $B=B^{\prime} \uparrow_{X\left(B^{\prime}\right)-N}$. Then $A \cong B$ and every clopen subset of $X(B)$ is of the form $[f=g]$ or $[f \neq g]$, and $X(B)^{*}$ is atomless iff for every $f$, $g \in B$ with $[f \neq g] \neq \varnothing$ there is an $h \in B$ with $\varnothing \neq[f \neq h] \subsetneq[f \neq g]$, and this is exactly what $\Pi$ asserts.

The next result was announced in [9].

THEOREM 9.13. Let $\Omega$ be an elementary class of algebras with a model-complete theory and let $T=\operatorname{Th}\left(\mathbf{V}\left(\Omega^{t}\right)\right)$. If $\Sigma_{0}$, respectively $\Sigma_{1}$, are axiom systems for $T$, respectively $\mathrm{Th}(\Omega)$, where we assume the sentences in $\Sigma_{1}$ are $\forall \exists$, then the following hold.

(a) The algebraically closed models of $T$ form the elementary class $\mathbf{I}{ }^{a}\left(\Omega_{+}^{t}\right)$, and it is axiomatized by $\boldsymbol{\Sigma}_{0} \cup\left(\boldsymbol{\Sigma}_{1}\right)^{*}$.

(b) If no member of $\Re$ properly contains a one-element subalgebra then the existentially closed models of $T$ form the elementary class $\left(\mathrm{I}_{0}^{e}\left(\mathfrak{\Omega}^{t}\right)\right)_{+}$, and the model companion of $T$ is axiomatized by $\Sigma_{0} \cup\left(\Sigma_{1}\right)^{*} \cup\{\Pi\}$. 
(c) If some member of $\Re$ properly contains a one-dlement subalgebra then the existentially closed models of $T$ form the elementary class consisting of those $A \in \mathbf{I} \Gamma_{0}^{a}\left(\Omega_{+}^{t}\right)$ for which

(i) $A \vDash \chi$, and

(ii) if, given $L(t)$-terms $p(\vec{u}, \vec{v})$ and $q(\vec{u}, \vec{v})$, there is a $B^{t} \in \mathbb{\Re}^{t}$ which has a one-element subalgebra $\{b\}$ for which $B^{t} \vDash \exists \vec{u}[p(\vec{u}, \vec{b}) \neq q(\vec{u}, \vec{b})], \vec{b}$ being $a$ sequence of $b$ 's, then we require that $A$ satisfy $\forall \vec{v} \exists \vec{u}[p(\bar{u}, \vec{v}) \neq q(\vec{u}, \vec{v})]$.

The model companion of $T$ is axiomatized by $\Sigma_{0} \cup\left(\Sigma_{1}\right)^{*} \cup\{\exists u \exists v(u \neq v)$, $\Pi, \chi\} \cup\{\forall \vec{v} \exists \vec{u}[p(\vec{u}, \vec{v}) \neq q(\vec{u}, \vec{v})] \mid p, q$ are as in (ii) $\}$.

If the language $L$ has only finitely many operational symbols, if each member of $\Re$ has at most one one-element subalgebra, and if $\mathrm{Th}(\Re)$ is complete then one can drop condition (ii) from part (c) as well as the corresponding axioms of the model companion.

(d) To obtain the algebraically and existentially closed models of $T \cup$ $\{\exists u \exists v(u \neq v)\}$ just add the requirement $\exists u \exists v(u \neq v)$ to the conditions in (a) and $(\mathrm{b})$.

Proof. (a) By Theorems 2.1 and 9.7(a) we know V( $\left(\Re^{t}\right)=\operatorname{IS\Gamma }^{a}\left(\Omega_{+}^{t}\right)$, and from Corollary $9.5 \mathbf{I} \Gamma^{a}\left(\Omega_{+}^{t}\right)$ is an elementary class whose theory is axiomatized by $\Sigma_{0} \cup\left(\Sigma_{1}\right)^{*}$. The axioms $\boldsymbol{\Sigma}_{1}^{*}$ are $\forall \exists$-positive, hence every algebraically closed model of $T$ must be in $\mathbf{I} \Gamma^{a}\left({\Omega_{+}^{t}}_{+}\right)$. On the other hand, if $A \in \Gamma^{a}\left(\Omega_{+}^{t}\right)$, $\Phi(\vec{v}) \in P_{n}^{+}, \vec{f} \in A$, and $A$ is a subalgebra of $B$ where $B=T$ and $B=\Phi(f)$, then we can assume $B \in \mathbf{I} \Gamma^{a}\left(\Omega_{+}^{t}\right)$, say $\alpha: B \rightarrow B^{\prime}$ is an isomorphism and $B^{\prime} \in \Gamma^{a}\left(\Omega_{+}^{\prime}\right)$. We want to show that $[\Phi(\vec{f})]_{A}=X(A)$ (see Lemma 9.3). If $\# A_{x}=1$ then $x \in[\Phi(\vec{f})]$ as $\Phi(\vec{f})$ is positive, so assume \# $A_{x}>1$. By Lemma 9.10 there is a $y \in X\left(B^{\prime}\right)$ such that the mapping $\beta: A_{x} \rightarrow B_{y}$ defined by $\beta(f x)=(\alpha f)(y)$ is an embedding. Since $[\Phi(\alpha \vec{f})]=X\left(B^{\prime}\right)$ (by Lemma 9.3) we have $B_{y}^{\prime}=\Phi((\alpha \vec{f})(y))$, and as $\operatorname{Th}\left(\Re^{t}\right)$ is model-complete and $A_{x}, B_{y}^{\prime} \in \Re^{t}$ it follows that $\alpha\left(A_{x}\right) \vDash \Phi((\alpha \vec{f})(y))$, hence $A_{x} \vDash \Phi(\vec{f}(x))$.

(b) In this case $\mathbf{V}\left(\Omega^{t}\right)=\left(I \Gamma^{a} \mathbf{S}\left(\Re^{t}\right)\right)_{+}$by Theorem $9.7(\mathrm{~b})$, and then by Theorem 2.1(d) and Corollary 7.5 V( $\left(\mathbb{\Omega}^{t}\right)=\left(\operatorname{IST}_{0}^{a}\left(\Re^{t}\right)\right)_{+}$. From Lemma 9.12 $\left(\mathbf{I} \Gamma_{0}^{a}\left(\Omega^{t}\right)\right)_{+}=\left\{A \in\left(I \Gamma^{a}\left(\Omega^{t}\right)\right)_{+} \mid A \vDash \Pi\right\}$, hence $\left(\mathbf{I} \Gamma_{0}^{a}\left(\Omega^{t}\right)\right)_{+}$is an elementary class axiomatized by $\boldsymbol{\Sigma}_{0} \cup\left(\boldsymbol{\Sigma}_{1}\right)^{*} \cup\{\Pi\}$. As the axioms in $\left(\boldsymbol{\Sigma}_{1}\right)^{*} \cup\{\Pi\}$ are $\forall \exists$ it follows that every existentially closed model of $T$ is in $\left(\mathbf{I}_{0}^{a}\left(\Omega^{t}\right)\right)_{+}$, and this is $\left(I \Gamma_{0}^{e}\left(\Omega^{t}\right)\right)_{+}$by Lemma 9.8(a), (b).

If $\# A=1$ and $A$ is a subalgebra of $B, B \in \mathrm{V}\left(\Re^{l}\right)$, then by Lemma 9.7(b), $\# B=1$, hence $A$ is existentially closed in $T$. So suppose $A \in \Gamma_{0}^{e}\left(\Omega^{t}\right)$, $\# A>1, \Phi(\vec{v}) \in P_{n}, \vec{f} \in A$ and $A$ is a subalgebra of $B$ where $B \vDash T$ and $B \vDash \Phi(\vec{f})$. Then we can assume $B \in \mathbf{I}_{0}^{e}\left(\Omega^{t}\right)$, say $\alpha: B \rightarrow B^{\prime}$ is an isomorphism and $B^{\prime} \in \Gamma_{0}^{e}\left(\Omega^{\prime}\right)$. Let us suppose $\Phi(\vec{v})$ is $\exists \vec{u}\left[\Phi_{0}(\vec{u}, \vec{v}) \& \&_{1 \leqslant i \leqslant n} p_{i}(\vec{u}, \vec{v})\right.$ $\neq q_{i}(\vec{u}, \vec{v})$ ], where $\Phi_{0}(\vec{u}, \vec{v})$ is a conjunct of atomic formulas. By Lemma 8.2 and part (a) above we only need to show $\left[\exists \vec{u}\left[\Phi_{0}(\vec{u}, \vec{f}) \& p_{i}(\vec{u}, \vec{f}) \neq q_{i}(\vec{u}, \vec{f})\right]\right]_{A}$ 
$\neq \varnothing$, for $1 \leqslant i \leqslant n$. Now since $B^{\prime} \vDash \Phi(\alpha \vec{f})$ it is possible to choose $y_{i} \in$ $X\left(B^{\prime}\right)$ such that $y_{i} \in\left[\exists \vec{u}\left[\Phi_{0}(\vec{u}, \alpha \vec{f}) \& p_{i}(\vec{u}, \alpha \vec{f}) \neq q_{i}(\vec{u}, \alpha \vec{f})\right]\right]$ for $1 \leqslant i \leqslant n$. Clearly this implies \# $B_{y_{i}^{\prime}}^{\prime}>1$. Let $\theta_{i}$ be the restriction of the congruence $\theta_{\left\{y_{i}\right\}}$ on $B^{\prime}$ to $\alpha(A)$. Then $\alpha(A) / \theta_{i} \in \mathbf{I S}\left(B_{y_{i}}^{\prime}\right)$ by Lemma 9.6(c), and as $B_{y_{i}}$ has no one-element subalgebras it follows that $\# \alpha(A) / \theta_{i}>1$, so $\alpha(A) / \theta_{i}$ is a simple algebra (all members of $\mathbf{S}\left(\Omega^{l}\right)$ with at least $\mathrm{t}$ vo elements are simple). Let $x_{i} \in X(A)$ be such that $\theta_{i}=\left\{\langle\alpha f, \alpha g\rangle \mid\langle f, g\rangle \in \hat{b}_{\left\{x_{i}\right\}}\right\}$ (such must exist by Lemma 9.6 as $\theta_{i}$ is a maximal congruence on $\left.\alpha(A)\right)$. Then the mapping $\beta$ : $A_{x_{i}} \rightarrow B_{y_{i}}^{\prime}$ defined by $\beta\left(f\left(x_{i}\right)\right)=(\alpha f)\left(y_{i}\right), f \in A$, is an embedding, and since $A_{x_{i}}$ and $B_{y_{i}}^{\prime}$ are members of $\Omega^{t}$, which has a model-complete theory, it follows that $x_{i} \in\left[\exists \vec{u}\left[\Phi_{0}(\vec{u}, \vec{f}) \& p_{i}(\vec{u}, \vec{f}) \neq q_{i}(\vec{u}, \vec{f})\right]\right]$.

(c) By Theorem 2.1(d), Theorem 9.7(a) and Corollary 7.5 we have $\mathbf{V}\left(\AA^{t}\right)=$ IS工 $\Gamma_{0}^{a}\left(\Omega_{+}^{t}\right)$. From Lemma 9.12 we have $\mathbf{I} \Gamma_{0}^{a}\left(\Omega_{+}^{t}\right)=\left\{A \in \mathbf{I} \Gamma^{a}\left(\Omega_{+}^{t}\right) \mid A \vDash \Pi\right\}$. Let $\mathfrak{\Omega}_{1}=\left\{A \in \mathbf{I} \Gamma_{0}^{a}\left(\Omega_{+}^{t}\right) \mid \# A>1, A \vDash \chi\right\}$, and let $B \in \mathfrak{R}^{t}$ be such that $\# B>1$ and $B$ has a one-element subalgebra. Select a fixed element $c$ from the Cantor discontinuum $C$ and define $\tilde{B}$ to be the subalgebra of $B[C]^{*}$ with universe $\left\{f \in B[C]^{*} \mid f(c)=b\right\}$, where $b$ is a particular one-element subalgebra of $B$. Then for $A$ in $\Gamma_{0}^{a}\left(\Omega_{+}^{t}\right), A \times \tilde{B} \in \Omega_{1}$, and as $A$ can be embedded in $A \times \tilde{B}$ it follows that $\mathbf{V}\left(\Omega^{t}\right)=\mathbf{S}\left(\Omega_{1}\right)$. Next let $\Omega_{2}$ be the class of all $A$ in $\Omega_{1}$ such that $A=\forall \vec{v} \exists \vec{u}[p(\vec{u}, \vec{v}) \neq q(\vec{u}, \vec{v})]$ for those $p, q$ required in condition (ii). For each such $p, q$ choose an algebra $B_{p, q}$ from $\Re^{t}$ which has the desired property in condition (ii). Then let $\tilde{B}_{p, q}$ be the subalgebra of $B_{p, q}[C]^{*}$ with universe $\left\{f \in B_{p, q}[C]^{*} \mid f(c)=b\right\}$, where $c$ is as above and $\{b\}$ is the particular one-element subalgebra of $B_{p, q}$ for $p$ and $q$. Then, for $A \in \Omega_{1}$, the algebra $A \times \Pi_{p, q} \tilde{B}_{p, q}$ is in $\Omega_{2}$, and $A$ can be embedded into this algebra. Thus $\Omega_{2}$ is an elementary class (by Corollary 9.5 ) which is axiomatized by the proposed axioms for the model companion of $T$, and such that $\mathbf{V}\left(\Omega^{t}\right)=$ $\mathbf{S}\left(\Omega_{2}\right)$. Since $\operatorname{Th}\left(\Omega_{2}\right)$ is axiomatizable by $\forall \exists$ sentences it follows that every existentially closed model of $T$ must be in $\Omega_{2}$.

On the other hand suppose $A \in \Gamma_{0}^{a}\left(\Omega_{+}^{t}\right)$ is in $\Omega_{2}$, and that $\Phi(\vec{v}) \in P_{n}$, $\vec{f} \in A$, and $A$ is a subalgebra of $B$ where $B \vDash T$ and $B \vDash \Phi(\vec{f})$. Then we can assume $B \in \Re_{2}$, and $\alpha: B \rightarrow B^{\prime}$ is an isomorphism with $B^{\prime} \in \Gamma_{0}^{a}\left(\Omega_{+}^{\prime}\right)$. Let us assume $\Phi(\vec{v})$ is as in part (b). By Lemma 8.2 and part (a) above we only need to show $\left[\exists \vec{u}\left[\Phi_{0}(\vec{u}, \vec{f}) \& p_{i}(\vec{u}, \vec{f}) \neq q_{i}(\vec{u}, \vec{f})\right]\right]_{A} \neq \varnothing$ for $1 \leqslant i \leqslant n$. Since $B^{\prime} \vDash$ $\Phi(\alpha \vec{f})$ we can choose $y_{i} \in X\left(B^{\prime}\right)$ with the property described in (b), for $1 \leqslant i \leqslant n$. Let $\theta_{i}$ be as defined in (b). If $\# \alpha(A) / \theta_{i}>1$ then proceed exactly as in (b) to the desired conclusion. However, if $\# \alpha(A) / \theta_{i}=1$ then the parameters $\alpha(\vec{f}) / \theta_{\left\{y_{i}\right\}}$ lie in a one-element subalgebra of $B^{\prime} / \theta_{\left\{y_{i}\right\}}$. Using Lemma 9.1 find $L(t)$-terms $r_{i}, \quad s_{i}$ such that $\Omega^{t} \models\left[r_{i}(\vec{u}, \vec{v}) \neq s_{i}(\vec{u}, \vec{v})\right] \leftrightarrow$ $\left[\Phi_{0}(\vec{u}, \vec{v}) \& p_{i}(\vec{u}, \vec{v}) \neq q_{i}(\vec{u}, \vec{v})\right]$. Then $B^{\prime} / \theta_{\left\{y_{i}\right\}}=\exists \vec{u}\left[r_{i}\left(\vec{u}, \alpha(\vec{f}) / \theta_{\left(y_{i}\right\}}\right) \neq\right.$ $s_{i}\left(\vec{u}, \alpha(\vec{f}) / \theta_{\left\{y_{i}\right)}\right)$, hence by (ii) we will have required that $\AA_{2}$, hence $A$, satisfy 
the sentence $\forall \vec{v} \exists \vec{u}\left[r_{i}(\vec{u}, \vec{v}) \neq s_{i}(\vec{u}, \vec{v})\right]$, so $\left[\exists \vec{u}\left[\Phi_{0}(\vec{u}, \vec{f}) \& p_{i}(\vec{u}, \vec{f}) \neq q_{i}(\vec{u}, \vec{f})\right]\right]_{A}$ $\neq \varnothing$, as desired.

Suppose now that the language has only finitely many operation symbols $\mathbf{f}_{1}, \ldots, \mathbf{f}_{m}$, that each member of $\Re$ has at most one one-element subalgebra, and that $\operatorname{Th}(\Re)$, hence $\operatorname{Th}\left(\Omega^{t}\right)$ is complete. If $p, q$ and $B$ are as in (ii) then

$$
\begin{aligned}
\mathfrak{R}^{t} \vDash \forall z[(\underset{1<i<m}{\&} & \left.\mathbf{f}_{i}(z, \ldots, z)=z\right) . \\
& \rightarrow \exists \vec{u}(p(\vec{u}, z, \ldots, z) \neq q(\vec{u}, z, \ldots, z))] .
\end{aligned}
$$

Thus given the situation $\# \alpha(A) / \theta_{i}=1$ above it follows that

$$
\begin{aligned}
\mathscr{\Omega}^{t} \vDash \forall z\left[\left(\underset{1<i<m}{\&} \mathbf{f}_{i}(z, \ldots, z)=z\right)\right. & \\
& \left.\rightarrow \exists \vec{u}\left(r_{i}(\vec{u}, z, \ldots, z) \neq s_{i}(\vec{u}, z, \ldots, z)\right)\right] .
\end{aligned}
$$

Now, looking at $A$, as $\left[\mathbb{Q}_{1 \leqslant i \leqslant m ; 1 \leqslant j \leqslant n} \mathbf{f}_{i}\left(f_{j}, \ldots, f_{j}\right)=f_{j}\right]$ is a clopen subset of $X(A)$, and as $[\forall u \forall v(u=v)]$ is not clopen, there must be an $x_{i}$ in the former, but not in the latter subset. Hence, as $A_{x_{i}} \in \Re^{t}, A_{x_{i}} \vDash \exists \vec{u}\left[r_{i}(\vec{u}, \vec{f}) \neq s_{i}(\vec{u}, \vec{f})\right]$, and thus $A_{x_{i}}$ must also satisfy $\exists \vec{u}\left[\Phi_{0}(\vec{u}, \vec{f}) \& p_{i}(\vec{u}, \vec{f}) \neq q_{i}(\vec{u}, \vec{f})\right]$.

(d) This is quite easy to check.

10. Discriminator formulas and universal Horn classes. We are going to generalize the results of $\S 9$ by working with a discriminator formula rather than a discriminator function. This will lead to the most general results we know of for constructing model-complete theories via the operator $\Gamma_{0}^{a}$. If $A$ is an $L(t)$-structure let $\operatorname{Red}_{t}(A)$ be the reduct of $A$ to an $L$-structure, and if $\Re$ is a class of $L(t)$-structures let $\operatorname{Red}_{t}(\Re)=\left\{\operatorname{Red}_{t}(A) \mid A \in \Re\right\}$. A formula $\tau \in$ $P_{4}^{+}$is a discriminator formula for a class $\Re$ if $\AA^{t} \vDash \tau(u, v, w, z) \leftrightarrow t(u, v$, $w)=z$.

Lemma 10.1. For any $\Re, \Gamma^{a}(\Re)=\operatorname{Red}_{t} \Gamma^{a}\left(\Omega^{t}\right)$.

Proof. Clearly every member of the right-hand side is in the left-hand side. So suppose $A \in \Gamma^{a}(\Re)$, and let $f, g, h \in A$. Then defining $k$ to be $h \uparrow_{[f=g]} \cup$ $f\left\lceil_{[f \neq g]}\right.$ it is immediate from the Patchwork Property that $k \in A$ and that $A_{x}^{t} \vDash t(f(x), \quad g(x), h(x))=k(x)$, for $x \in X(A)$, hence the subset of $\mathrm{II}_{x \in X(A)} A_{x}^{t}$ consisting of the universe of $A$ is closed under the operation $t$, and thus this subset is a subuniverse corresponding to a substructure $A^{\prime}$ with $\operatorname{Red}_{t}\left(A^{\prime}\right)=A$.

For $\tau \in P_{4}^{+}$let us define $\tau^{e}$ to be the sentence: $\forall u \forall v \forall w \exists z \tau(u, v, w, z)$.

LEMMA 10.2. Let $\tau$ be a discriminator formula for $\Re$. Then

$$
\left\{A \in \mathbf{S \Gamma}^{a}(\Re) \mid A \vDash \tau^{e}\right\} \subseteq \operatorname{Red}_{t} \mathbf{S}^{a}\left(\Re^{t}\right) .
$$


Proof. Let $A$ be a substructure of $B, B \in \Gamma^{a}(\Re)$ and suppose $A \vDash \tau^{e}$. Let $B^{\prime} \in \Gamma^{a}\left(\Omega^{t}\right)$ be such that $B=\operatorname{Red}_{t}\left(B^{\prime}\right)$. If $f, g, h \in A$ choose $k \in A$ such that $A \vDash \tau(f, g, h, k)$. But then $B^{\prime} \vDash \tau(f, g, h, k)$ as $\tau$ is an existential formula, and then, by Lemma $9.3, B^{\prime} \vDash t(f, g, h)=k$, hence $A$ is a reduct of a substructure $A^{\prime}$ of $B^{\prime}$.

LEMMA 10.3. If $\tau$ is a discriminator formula for $\Re$ then

$$
\left\{A^{\prime} \in \mathbf{S \Gamma}^{a}\left(\Omega^{t}\right) \mid A^{\prime} \vDash \tau^{e}\right\} \vDash \tau(u, v, w, z) \leftrightarrow t(u, v, w)=z .
$$

Proof. This follows from Lemma 9.3, arguing as in Lemma 10.2.

For $\tau \in P_{4}^{+}$let us define a translation $\#_{\tau}$ from $L(t)$-formulas to $L$-formulas as follows. First $\#_{\tau}(t(u, v, w)=z)$ is $\tau(u, v, w, z)$ if $u, v, w, z$ are variables, and $\#_{\tau}(\alpha)$ is just $\alpha$ if $\alpha$ is any atomic formula not involving the function symbol $t$. Then, given any $L(t)$-formula $\Phi$ first replace it by a logically equivalent formula of the form $\overrightarrow{Q u}\left[\bigvee_{i} \wedge_{j} \pm \alpha_{i, j}\right]$ where each $\alpha_{i, j}$ is an atomic formula, and indeed of the form $t(u, v, w)=z$ with $u, v, w, z$ variables if $\alpha_{i, j}$ involves the function symbol $t$. Then define $\#_{\tau}(\Phi)$ to be $\overrightarrow{Q u}\left[\bigvee_{i} \wedge_{j} \pm \#_{\tau}\left(\alpha_{i, j}\right)\right]$. With this translation the following is immediate from Lemma 10.3 .

LEMMA 10.4. Suppose $\tau$ is a discriminator formula for $\Re, A^{\prime} \in \mathbf{S} \Gamma^{a}\left(\Re^{t}\right)$, $A^{\prime} \vDash \tau^{e}$, and that $\Phi$ is an $L(t)$-sentence. Then $A^{\prime} \vDash \Phi$ iff $A^{\prime} \vDash \#_{\tau}(\Phi)$.

LEMMA 10.5. If $\Re$ is an elementary class, $\tau$ a discriminator formula for $\Re, \boldsymbol{\Sigma}^{0}$ a set of axioms for $\operatorname{Th}(\mathbf{S P}(\Re))$, and $\mathfrak{Q}$ an elementary subclass of $\mathbf{I}^{a}\left(\Omega^{t}\right)$ axiomatized by $\boldsymbol{\Sigma}^{1}$, then $\operatorname{Red}_{t}(\mathfrak{g})$ is an elementary class axiomatized by $\boldsymbol{\Sigma}^{0} \cup \#_{\tau}\left(\boldsymbol{\Sigma}^{1}\right)$ $\cup\left\{\tau^{e}\right\}$.

Proof. Since $\Gamma^{a}\left(\Omega^{t}\right) \vDash \tau(u, v, w, z) \leftrightarrow t(u, v, w)=z$ it is clear that $\mathfrak{Q}$, hence $\operatorname{Red}_{t}(\mathfrak{Q})$ satisfies the axioms mentioned. On the other hand, if $A$ is an $L$-structure satisfying $\Sigma^{0}$, then, by Theorem $2.1(\mathrm{~d})$, we can assume $A \in$ $\mathrm{S \Gamma}^{a}(\Re)$. Then from $A \vDash \tau^{e}$ one concludes by Lemma 10.2 that $A=\operatorname{Red}_{t}\left(A^{\prime}\right)$ for some $A^{\prime}$ in $\mathbf{S} \Gamma^{a}\left(\Omega^{t}\right)$. Then if $A \vDash \#_{\tau}\left(\Sigma^{1}\right)$ it follows from Lemma 10.4 that $A^{\prime} \vDash \Sigma^{1}$, hence $A \in \operatorname{Red}_{t}(\mathfrak{Q})$.

Lemma 10.6. Suppose $\tau$ is a discriminator formula for $\Re$ and that $A^{\prime}$, $B^{\prime} \in \Gamma^{a}\left(\Omega^{t}\right)$. Let $A=\operatorname{Red}_{t}\left(A^{\prime}\right)$ and $B=\operatorname{Red}_{t}\left(B^{\prime}\right)$. Then if $\alpha: A \rightarrow B$ is an embedding one also has $\alpha: A^{\prime} \rightarrow B^{\prime}$ is an embedding.

Proof. Suppose $f, g, h, k \in A^{\prime}$ and $A^{\prime} \vDash t(f, g, h)=k$. Then $A^{\prime} \vDash$ $\tau(f, g, h, k)$, hence $B^{\prime} \vDash \tau(\alpha f, \alpha g, \alpha h, \alpha k)$ as $\tau$ is an existential formula. But then $B^{\prime} \vDash t(\alpha f, \alpha g, \alpha h)=\alpha k$, hence $\alpha$ preserves the operation $t$.

Our main result is an obvious copy of Theorem 9.13. 
THEOREM 10.7. Let $\Re$ be an elementary class of algebras with a model-complete theory and suppose $\tau$ is a discriminator formula for $\Re$. Let $T=$ $\operatorname{Th}\left(\mathbf{S P}\left(\Re_{+}\right)\right)$, let $\Sigma^{0}$ be a set of axioms for $T$, and let $\boldsymbol{\Sigma}_{1}$ be a set of $\forall \exists$ axioms for $\operatorname{Th}(\Re)$.

(a) The algebraically closed models of $T$ form the elementary class $I \Gamma^{a}\left(\Omega_{+}\right)$, and it is axiomatized by $\boldsymbol{\Sigma}^{0} \cup \#_{\tau}\left(\boldsymbol{\Sigma}_{1}^{*}\right) \cup\left\{\tau^{e}\right\}$.

(b) If no member of $\Re$ properly contains a one-element subalgebra then the existentially closed models of $T$ form the elementary class $\left(\mathrm{Ir}_{0}^{e}(\Omega)\right)_{+}$, and the model companion of $T$ is axiomatized by $\boldsymbol{\Sigma}^{0} \cup \#_{\tau}\left(\boldsymbol{\Sigma}_{1}^{*} \cup\{\Pi\}\right) \cup\left\{\tau^{e}\right\}$.

(c) If some member of $\Re$ properly contains a one-element subalgebra then the existentially closed models of $T$ form the elementary class consisting of those $A \in \mathbf{I} \Gamma_{0}^{a}\left(\Omega_{+}\right)$for which

(i) $A \vDash \#_{\tau}(\chi)$, and

(ii) if, given $L(t)$-terms $p(\vec{u}, \vec{v})$ and $q(\vec{u}, \vec{v})$, there is a $B^{t} \in \Omega^{t}$ which has a one-element subalgebra of $\{b\}$ for which $B^{t} \vDash \exists \vec{u}[p(\vec{u}, \vec{b}) \neq q(\vec{u}, \vec{b})], \vec{b}$ being $a$ sequence of $b$ 's, then we require that $A$ satisfy $\#_{\tau}[\forall \vec{v} \exists \vec{u}(p(\vec{u}, \vec{v}) \neq q)(\vec{u}, \vec{v})]$.

The model companion of $T$ is axiomatized by $\Sigma^{0} \cup \#_{\tau}\left(\Sigma_{1}^{*} \cup\{\Pi, \chi\}\right) \cup$ $\#_{\tau}\left(\boldsymbol{\Sigma}^{\prime}\right) \cup\left\{\exists u \exists v(u \neq v), \tau^{e}\right\}$, where $\boldsymbol{\Sigma}^{\prime}$ is the set of all formulas $\forall \vec{v} \exists \vec{u}[p(\vec{u}, \vec{v})$ $\neq q(\vec{u}, \vec{v})]$ with $p, q$ being as in (ii).

If the language $L$ has only finitely many operational symbols, if each member of $\Re$ has at most one one-element subalgebra, and if $\mathrm{Th}(\Omega)$ is complete then one can drop condition (ii) from part (c) as well as the corresponding axioms of the model companion.

(d) To obtain the algebraically and existentially closed models of $T \cup$ $\{\exists u \exists v(u \neq v)\}$ just add the requirement $\exists u \exists v(u \neq v)$ to the conditions in (a) and $(\mathrm{b})$.

Proof. First note that in each of (a), (b), and (c) the classes described are such that every member of $\mathbf{S P}\left(\Re_{+}\right)$can be embedded in a member of them (use Theorem 9.13 and Lemma 10.1). From Lemma 10.5 one sees that these classes are elementary. The axioms presented for these classes are those given in Lemma 10.5 with the exception of $\#_{\tau}\left(\boldsymbol{\Sigma}_{0}\right)$, where $\boldsymbol{\Sigma}_{0}$ is a set of axioms for $\operatorname{Th}\left(\mathbf{S P}\left(\Omega_{+}^{t}\right)\right)$; however this is no problem as we can deduce $\#_{\tau}\left(\boldsymbol{\Sigma}_{0}\right)$ from $\Sigma^{0} \cup\left\{\tau^{e}\right\}$ by Lemmas 10.2 and 10.4.

So we only have to verify that the above classes are indeed the algebraically closed and existentially closed models of $T$. For (a) note that the axioms $\#{ }_{\tau}\left(\Sigma_{1}\right) \cup\left\{\tau^{e}\right\}$ are $\forall \exists$-positive sentences, hence every algebraically closed model of $T$ must satisfy them. On the other hand the embeddings between members of $\mathbf{I} \Gamma^{a}\left(\Omega_{+}\right)$are precisely those between the corresponding members (see Lemma 10.1) of $I \Gamma^{a}\left(\Omega_{+}^{t}\right)$ by Lemma 10.6, and thus by Theorem 9.13 it is clear that the members of $\mathbf{I} \Gamma^{a}\left(\Omega_{+}^{t}\right)$ must be algebraically closed in $T$. For (b) and (c) use the correspondence of embeddings, as in (a), to see that the 
classes are model-complete. Part (d) is again straightforward.

Lipschitz and Saracino [26] were the first to use sheaf constructions to find the model companion of a theory. Subsequently this was generalized by MacIntyre [27] using the notion of a positively model-complete theory, namely a theory $T$ such that for every $\Phi \in E_{n}$ there is a $\Psi \in E_{n}^{+}$such that $T \vdash \neg \Phi \leftrightarrow \Psi$. (Note that a theory $T$ is positively model-complete iff it is model-complete and for every $\Phi$ in $E_{n}$ there is a $\Psi$ in $E_{n}^{+}$such that $T \vdash \Phi \leftrightarrow \Psi$.) An obvious limitation of this condition is that if $\Re$ is the class of models of a positively model-complete theory of algebras then no member of $\Re$ can properly contain a one-element subalgebra because there is a formula $\Phi \in E_{2}^{+}$such that $\Omega \vDash u \neq v \leftrightarrow \Phi(u, v)$. Later Comer [14] noted that Macintyre's conditions could be considerably simplified, and now we will show that Comer's theorem is a special case of Theorem 10.7(b), (d). For the time being we will only consider theories of algebras, and at the end of this section discuss some appropriate changes to handle languages with relation symbols.

COROllary 10.8 (COMER). Let T be a positively model-complete theory of algebras such that there are terms $p(u, v), s(u, v)$, an atomic formula $\Delta(u)$, and constants 0,1 such that

(i) $T \vdash \Delta(u) \leftrightarrow(u=0 \vee u=1)$,

(ii) $T \vdash \forall u[p(u, 1)=u \& p(u, 0)=0]$, and

(iii) for every model $A$ of $T$ the relativized reduct $\langle\{0,1\} ; p, s\rangle$ is a lattice.

Then, if $\Re$ is the class of models of $T$, it follows that $\mathrm{Th}\left(\Gamma_{0}^{e}(\Re)\right)$ is model-complete.

PROOF. First note that the formula $u_{1}=u_{2} \bigvee u_{3}=u_{4}$ is equivalent modulo $T$ to the primitive positive formula

$$
\begin{array}{r}
\exists v \exists w\left[\Delta(v) \& \Delta(w) \&\left(\underset{1<i<4}{\&} p\left(u_{i}, s(v, w)\right)=u_{i}\right)\right. \\
\left.\& p\left(u_{1}, v\right)=p\left(u_{2}, v\right) \& p\left(u_{3}, w\right)=p\left(u_{4}, w\right)\right]
\end{array}
$$

hence, as $T$ is positively model-complete, every formula of $E_{n}$ is equivalent modulo $T$ to a formula in $P_{n}^{+}$. In particular the formula $(u=v \& w=z) \bigvee$ $(u \neq v \& u=w)$ is equivalent to a formula $\tau$ in ${P_{4}}^{+}$, and this is a discriminator formula for the models of $T$. Thus the conclusion follows from Theorem 10.7(b), (d), and Theorem 4.5(b).

COROllary 10.9 (MACINTYRE). Let the language L contain operation symbols $+, \cdot,-, 0,1$ for the language of rings with unity, and suppose $\Re$ is a class of L-algebras such that

(i) $\operatorname{Th}(\Re)$ is positively model-complete, 
(ii) $\mathrm{Th}(\Re)$ includes axioms for nontrivial rings, and

(iii) $\mathrm{Th}(\Re)$ includes a statement which says "there are exactly two idempotent elements in the ring".

Then $\mathrm{Th}\left(\Gamma_{0}^{e}(\Re)\right)$ is model-complete.

For applications of Theorem 10.7 we have of course discriminator varieties (some of which were discussed in §6; this includes Comer's study of monadic algebras), the result of Lipschitz and Saracino which says that if $\Re$ is the class of algebraically closed fields in the language of rings with unity then $\operatorname{Th}\left(\Gamma_{0}^{e}(\Re)\right)$ is the model companion of the class of nontrivial commutative rings with unity and without nonzero nilpotent elements, and the result of Macintyre which says that if $\Omega$ is the class of linearly ordered real-closed fields in the language of $f$-rings with unity then $\operatorname{Th}\left(\Gamma_{0}^{e}(\Omega)\right)$ is the model companion of the class of nontrivial commutative $f$-rings with unity and no nonzero nilpotent elements. In the last two examples we have parallel results in the corresponding languages without unity by using Theorem 10.7(c), (d), namely we just delete the word 'unity' and replace $\Gamma_{0}^{e}(\Re)$ by the appropriate subclass of $\Gamma_{0}^{a}\left(\Omega_{+}\right)$as required by Theorem 10.7(c) (note that we can dispense with the condition (ii) of (c) in these two cases). Furthermore we can write down a system of axioms for the algebraically closed and existentially closed members of these classes, and give a reasonably good description of these members in terms of the $\Gamma$ operators. To justify our claim for parallel results it suffices to note that the following formula $\tau\left(v_{1}, \ldots, v_{4}\right)$ is a discriminator formula on any class of fields in the language $+, \cdot,-, 0$ of rings:

$$
\begin{gathered}
\exists u_{1} \exists u_{2} \exists u_{3}\left[\underset{1<i<3}{\&}\left(v_{i} \cdot u_{3}=v_{i}\right) \& u_{1} \cdot u_{2}=u_{3} \& u_{1}^{2}\right. \\
\cdot\left(v_{1}-v_{2}\right)^{2}=u_{1} \cdot\left(v_{1}-v_{2}\right) \\
\left.\& v_{4}=u_{1} \cdot v_{1} \cdot\left(v_{1}-v_{2}\right)+v_{3} \cdot\left(u_{3}-u_{1} \cdot\left(v_{1}-v_{2}\right)\right)\right] .
\end{gathered}
$$

Finally we look briefly at languages with relation symbols. The first complication is the number of possible one-element structures, so let us just consider the case that there are no one-element structures in $\mathbf{S}(\Re)$, where $\mathscr{\Re}$ is an elementary class with a model-complete theory, and $\tau$ is a discriminator formula for $\Re$. (This will cover the cases considered by Macintyre and Comer.) Then to achieve an analogue of Lemma 9.2 one needs rather strong assumptions, for example the following two will do:

(a) $\operatorname{Th}(\Re)$ is complete, and

(b) for each fundamental $n$-ary relation $\mathbf{r}$ there is a formula $\Psi_{r} \in E_{n}^{+}$such that $\Re \vDash \neg \mathbf{r}\left(v_{1}, \ldots, v_{n}\right) \leftrightarrow \Psi_{r}\left(v_{1}, \ldots, v_{n}\right)$. 
To see how these are used suppose $\Phi$ is an $\forall \exists$ sentence. First use (b) to put it in the form $\forall \exists \wedge \vee( \pm$ atomic), where the only negated atomic formulas are of the form $p \neq q$. Then use (a) to convert $\bigvee( \pm$ atomic) subformula into an $\exists \wedge( \pm$ atomic $)$ formula, where again the only negated atomic formulas are of the form $p \neq q$. Finally use the $t$ as in Lemma 9.2 to change the last sentence into an $\forall \exists \wedge$ (atomic) sentence $\Phi^{\prime}$ such that for $A \in \Re, A^{t} \vDash \Phi^{\prime}$ iff $A \vDash \Phi$. Then one has the conclusions of Theorem 10.7(a), (b), using $T=$ $\operatorname{Th}(\mathbf{S P}(\Re))$, replacing $\Omega_{+}$by $\Re$, and $\left(\mathbf{I} \Gamma_{0}^{e}(\Re)\right)_{+}$by $\mathbf{I} \Gamma_{0}^{e}(\Re)$, and using $\Sigma_{1}^{\prime}$ instead of $\Sigma_{1}^{*}$.

Appendix. A sheaf of $L$-structures is a triple $\tilde{S}=(S, X, \pi)$ where

(a) $S$ and $X$ are topological spaces,

(b) $\pi: S \rightarrow X$ is a surjective local homeomorphism,

(c) $S_{x}=: \pi^{-1}(x)$ is an $L$-structure (the stalk at $x$ ) for each $x \in X$,

(d) if $f$ is a fundamental $n$-ary operation and $S_{n}$ is the subspace $\Pi_{x \in X} S_{x}^{n}$ of $S^{n}$, then $\mathrm{f}: S_{n} \rightarrow S$ is a continuous map, and

(e) if $\mathbf{r}$ is a fundamental $n$-ary relation and $\chi_{r}$ is the characteristic function of $r$ mapping $S_{n}$ to the two-element discrete space 2 , then $\chi_{r}^{-1}(1)$ is open. ${ }^{12}$

A global section of $\tilde{S}$ is a continuous map $\sigma: X \rightarrow S$ such that $\sigma$ is the identity map on $X$. The structure of global sections $\gamma(\tilde{S})$ is the substructure of $\Pi_{x \in X} S_{x}$ whose universe consists of a the global sections of $\tilde{S}$.

LEMma 1. Let $\tilde{S}=(S, X, \pi)$ be a sheaf of L-structures and suppose $\sigma$ is an element of $\Pi_{x \in X} S_{x}$. Then $\sigma \in \gamma(\tilde{S})$ iff for each $x \in X$ there is an open subset $U_{x}$ of $S$ such that $\sigma(x) \in U_{x} \subseteq \sigma(X)$, and $\pi$ restricted to $U_{x}$ is a homeomorphism.

Proof. If for each $x \in X$ there is such a $U_{x}$ then given $x \in X$ and any open neighborhood $V$ of $\sigma(x)$ it follows that $\sigma^{-1}\left(V \cap U_{x}\right)=\pi\left(V \cap U_{x}\right)$, an open subset of $X$, so $\sigma$ is continuous. On the other hand if $\sigma$ is continuous then for $x \in X$ choose $V_{x}$ to be an open neighborhood of $\sigma(x)$ such that $\pi$ restricted to $V_{x}$ is a homeomorphism, and then let $U_{x}=\sigma \circ \sigma^{-1}\left(V_{x}\right) . U_{x}$ is open as it is a subset of $V_{x}$ and $\pi\left(U_{x}\right)=\sigma^{-1}\left(V_{x}\right)$, and clearly $\pi$ restricted to $U_{x}$ is a homeomorphism.

From this lemma it is easy to obtain the following basic properties of $\gamma(\tilde{S})$.

COROLLARY 2. For a sheaf of L-structures $\tilde{S}$ we have:

(a) global sections are open maps,

(b) if $\sigma, \tau \in \gamma(\tilde{S})$ then $\{x \in X \mid \sigma(x)=\tau(x)\}$ is open,

(c) if $\mathbf{r}$ is a fundamental $n$-ary relation and $\sigma_{1}, \ldots, \sigma_{n} \in \gamma(\tilde{S})$ then $\{x \in$ $\left.X \mid S_{x} \vDash \mathbf{r}\left(\sigma_{1}(x), \ldots, \sigma_{n}(x)\right)\right\}$ is open,

\footnotetext{
12 In [27] Macintyre has a stronger requirement, namely that $\chi_{r}$ be continuous.
} 
(d) if $N$ is a clopen subset of $X$ and $\sigma, \tau \in \gamma(\tilde{S})$ then $\sigma \uparrow_{N} \cup \tau \uparrow_{X-N}$ is also in $\gamma(\tilde{S})$, and

(e) if $X$ is compact and has a basis of clopen sets then $\gamma(\tilde{S})$ is a subdirect product of the $S_{x}, x \in X$.

In view of conditions (b) and (c) above we introduce the operator $\Gamma$ defined by the properties (1) and (3) of $\S 1$ plus:

(2) for $\Phi\left(u_{1}, \ldots, u_{m}\right)$ an atomic formula and $f_{1}, \ldots, f_{m} \in A$, $\left[\Phi\left(u_{1}, \ldots, u_{m}\right)\right]$ is an open subset of $X$.

This is our most general Boolean product operator, and next we show that it is equivalent to the formation of global sections of sheaves of $L$-structures over Boolean spaces.

TheOREM 3. (a) If $\tilde{S}=(S, X, \pi)$ is a sheaf of L-structures and $X$ is a Boolean space then $\gamma(\tilde{S}) \in \Gamma\left(\left\{S_{x} \mid x \in X\right\}\right)$.

(b) Suppose $A \in \Gamma(\Re)$. Let $S=\cup \Pi_{x \in X(A)} A_{x}$ have as a basis for its topology all sets $\{\langle x, f(x)\rangle \mid x \in N\}$ where $f \in A$ and $N \in X(A)^{*}$, let $X=$ $X(A)$, and define $\pi$ by $\pi(\langle x, a\rangle)=x$. Then $\tilde{S}=(S, X, \pi)$ is a sheaf (of $L$-structures) over a Boolean space and $\gamma(\tilde{S})$ is isomorphic to $A$ under the map $\alpha$ defined by $\alpha(\sigma)(x)=a$ if $\sigma(x)=\langle x, a\rangle$.

Proof. (a) This is an immediate consequence of Corollary 2.

(b) Clearly we have defined a sheaf of $L$-structures $\tilde{S}$. Then use Lemma 1 to verify that the global sections of $\tilde{S}$ are precisely those $\sigma$ corresponding to the members of $A$ under the mapping $\alpha$.

A sheaf $\tilde{S}=(S, X, \pi)$ is Hausdorff if $S$ is a Hausdorff space. It is an easy exercise to show that if $\mathbf{S}$ is a Hausdorff sheaf with $X$ a Boolean space then $\gamma(\tilde{S}) \in \Gamma^{a}\left(\left\{S_{x} \mid x \in X\right\}\right)$, and conversely, if $A \in \Gamma^{a}(\Omega)$ then the sheaf constructed in Theorem 3(b) is Hausdorff.

Added IN Proof. Carson [43] independently discovered the results attributed to Lipschitz and Saracino.

\section{REFERENCES}

1. R. F. Arens and I. Kaplansky, Topological representation of algebras, Trans. Amer. Math. Soc. 63 (1948), 457-481. MR 10, 7.

2. C. J. Ash, Reduced powers and Boolean extensions, J. London Math. Soc. 9 (1975), 429-432.

3. J. Baldwin and A. H. Lachlan, On universal Horn classes categorical in some infinite power, Algebra Universalis 3 (1973), 98-111. MR 50 \# 4273.

4. B. Banaschewski and E. Nelson, Boolean powers as algebras of continuous functions, 1975 (preprint).

5. S. Bulman-Fleming and H. Werner, Equational compactness in quasi-primal varieties, 1975 (preprint).

6. S. Burris, Boolean powers, Algebra Universalis 5 (1975), 341-360.

7. S. Burris and H. Werner, Sheaf constructions and their elementary properties. I, II, 1975 (preprints).

8. Decidable theories, Notices Amer. Math. Soc. 22 (1975), A-475. 
9. , Model companions for discriminator varieties, Notices Amer. Math. Soc. 23 (1976),

A-28.

10. C. C. Chang and H. J. Keisler, Model theory, North-Holland, Amsterdam, 1973.

11. S. D. Comer, Representations by algebras of sections over Boolean spaces, Pacific J. Math. 38 (1971), 29-38. MR 46 \#3412.

12. Elementary properties of structures of sections, Bol. Soc. Mat. Mexicana 19 (1974), 78-85.

13. Monadic algebras with finite degree, Algebra Universalis 5 (1975), 315-329.

14. Complete and model-complete theories of monadic algebras, Colloq. Math. 34 (1976), 183-190.

15. B. A. Davey, Sheaf spaces and sheaves of universal algebras, Math. Ż. 134 (1973), 275-290. MR 48 \#8345.

16. A. Ehrenfeucht, Decidability of the theory of one function, Notices Amer. Math. Soc. 6 (1959), 268

17. P. Eklof and G. Sabbagh, Model-completions and modules, Ann. Math. Logic $2(1970 / 71)$, no. 3, 251-295. MR 43 \#3105.

18. Ju. L. Eršov, Decidability of the elementary theory of relatively complemented lattices and of the theory of filters, Algebra i Logika Sem. 3 (1964), no. 3, 17-38. (Russian) MR 31 \#4725.

19. Elementary theory of Post varieties, Algebra i Logika 6 (1967), no. 5, 7-15. (Russian) MR 38 \# 34.

20. Ju. L. Eršov, I. A. Lavrov, A. D. Taimanov and M. A. Taiclin, Elementary theories, Russian Math. Surveys 20 (1965), 35-105. MR 32 \#4012.

21. S. Feferman and R. L. Vaught, The first order properties of products of algebraic systems, Fund. Math. 47 (1959), 57-103. MR 21 \#171.

22. P. R. Halmos, Lectures on Boolean algebras, Van Nostrand, Princeton, N.J., 1963. MR 29 \#4713.

23. L. Henkin, J. D. Monk and A. Tarski, Cylindric algebras. I, North-Holland, Amsterdam, 1971. MR 47 \#3171.

24. K. Keimel and H. Werner, Stone duality for varieties generated by quasi-primal algebras, Mem. Amer. Math. Soc. No. 148 (1974), 59-85. MR 50 \# 12861.

25. P. Lindström, On model-completeness, Theoria (Lund) 30 (1964), 183-196. MR 31 \#3317.

26. L. Lipschitz and D. Saracino, The model companion of the theory of commutative rings without nilpotent elements, Proc. Amer. Math. Soc. 38 (1973), 381-387.

27. A. Macintyre, Model-completeness for sheaves of structures, Fund. Math. 81 (1973/74), no. 1, 73-89. MR 49 \#2361.

28. J. A. Makowsky, On some conjectures connected with complete sentences, Fund. Math. 81 (1974), 193-202. MR 51 \#2894.

29. A. I. Mal'cev, The metamathematics of algebraic systems. Collected papers: 1936-1967, North-Holland, Amsterdam, 1971. MR 50 \# 1877.

30. R. S. Pierce, Modules over commutative regular rings, Mem. Amer. Math. Soc. No. 70 (1967). MR 36 \#151.

31. M. O. Rabin, Decidability of second-order theories and automata on infinite trees, Trans. Amer. Math. Soc. 141 (1969), 1-35. MR 40 \#30.

32. M. Rubin, Boolean algebras: Undecidability with distinguished subalgebra and interpretation in automorphism groups, Notices Amer. Math. Soc. 22 (1975), A-645.

33. D. Saracino, Model companions for $\boldsymbol{\aleph}_{0}$-categorical theories, Proc. Amer. Math. Soc. 39 (1973), 591-598. MR 47 \#4786.

34. W. Szmielew, Elementary properties of Abelian groups, Fund. Math. 41 (1955), 203-271. MR $17,233$.

35. A. Tarski, Arithmetical classes and types of Boolean algebras, Bull. Amer. Math. Soc. 55 (1949), 64.

36. W. Taylor, Pure compactifications in quasi-primal varieties, Canad. J. Math. 28 (1976), $50-62$. 
37. H. Volger, The Feferman-Vaught theorem revisited, 1975 (preprint).

38. J. Waszkiewicz and B. Weglorz, Some models of theories of reduced powers, Bull. Acad. Polon. Sci. Sér. Sci. Math. Astronom. Phys. 16 (1968), 683-685. MR 38 \# 5602.

39. , On $\omega_{0}$-categoricity of powers, Bull. Acad. Polon. Sci. Sér. Sci. Math. Astronom. Phys. 17 (1969), 195-199. MR 41 \#3262.

40. B. Weglorz, Substructures of reduced powers, Fund. Math. 89 (1975), 191-197.

41. V. Weispfennig, Model-completeness and elimination of quantifiers for subdirect products of structures, J. Algebra 36 (1975), 252-277.

42. H. Werner, Algebraic representation and model theoretic properties of algebras with the ternary discriminator, 1975 (preprint).

43. A. B. Carson, The model completion of the theory of commutative regular rings, J. Algebra 27 (1973), 136-146.

Department of Pure Mathematics, University of Waterloo, Waterloo, Ontario, CANADA N2L 3G1 (Current address of Stanley Burris)

Department of Mathematics, Technische Hochschule, Darmstadt, Federal Republic OF GERMANY

Current address (Heinrich Werner): Department of Mathematics, Gesamthochschule Kassel, Kassel, Federal Republic of Germany 